\section{Zur Theorie des Färbeprocesses in der Indigoktipe.}

Fünfte Mittheilung ') ũber Indigofärberei. Von A. Binz und F. Rung.

Zur Reduction des Indigos bei Gegenwart von Alkali eignet sich eine ganze Reihe ron Reagentien ${ }^{2}$ ). Nicht alle sind für technische Zwecke billig genug, aber immerhin hat man hier noch die Auswahl zwischen Zinkstaub, Ferrohydroxyd und Natriumhydrosulfit. Diese drei finden in der Baumwollfärberei Verwendung. Dazu kommt noch für den Kattundruck Traubenzucker. Das Küpen erfolgt in einfacher Weise durch Immersion der Stoffe in der Kälte.

Angesichts dieser Mannigfaltigkeit der Methoden und der Leichtigkeit ihrer Handhabung in der Baumwollfärberei ist es auffallend, dass keine von ihnen für das Küpen von Wolle die herrschende ist. Die kalten Baumwollkūpen versagen hier, man muss warme Flotten herstellen ${ }^{3}$ ). Das gelingt zwar in besonders reinlicher und glatter Weise mit Natriumhydrosulfit"), und man sollte darum annehmen, dass dieses ausgezeichnete Verfahren den Färbern genüge. Das ist aber merkwürdiger Weise nicht der Fall. Man zieht ihm vielmehr ein anderes, uraltes vor, das unleugbare Mängel zeigt und recht wenig zum Rüstzeug der modernen Technik passt: die Gährungsküpe.

Caesar erzählt, dass die Britannier sich mit Waid blau färbten, und diese von den Barbaren der Vorzeit stammende Methode ${ }^{5}$ )

1) Im Auszug vorgetragen von A. Binz. - Die früheren Mittheilungen s. diese Zeitschr. 1898, 957; 1899,$515 ; 1900,412 ; 1902,557$.

y) v. Georgievics („Der Indigo“ 1892, S. 146) zăhlt deren 22 anf, dazu noch die Gähruugsmittel.

3) Es ist sehr merkwūrdig, dass Wolle warm gekūpt werden muss, Baumwolle dagegen nicht. Da Wolle bekanntlich die grōssere Verwandtschaft zu Farbstoffen hat, so sollte man voraussetzen, dass ein Färbeprocess, der in der Baumwollkūpe in der Kälte vor sich geht, für Wolle erst recht nicht durch Erwärmen unterstūtzt zu werden brauchte. Dass das Umgekehrte der Fall ist, lässt sich vielleicht aus einer Auffassung des Küpprocesses orklären, die wir weiter unten mittheilen wollen.

4) Schützenberger und Lalande, Chem. Centralbl. 1873, 735.

5) Die heutigo Găhrungskūpe hat sich aus der Waidküpe entwickelt. (Vergl. die historischen Notizen im Indigobuch der Badischen Anilinund Sodafabrik, Ludwigshafen 1900, S. 125. S. auch 0 . N. Witt, Chem. Technologie d. Gespinstfasern 1888 , S. 15 u. 24.) - Herr Geh.-Rath Prof. Dr. Bü cheler in Bonn, den wir vor längerer Zeit (1899) um Auskunft über das Alter der Waidküpe ersuchten, wies uns auf die betreffende Stelle in Caesar de bello gallico $\mathrm{V}, 14$ hin: Omnes se Britanni vitro inficinnt, quod caeruleam efficit colorem, atque hoc horridiores sunt in pugna aspectu. wendet man heutzutage noch im Princip bei der Gährungsküpe an. Sie wird mit Krapp, Kleie, Waid, Syrup, Kalk und Soda angesetzt und hat den Vorzug der Billigkeit.

Dem stehen aber grosse Nachtheile gegenüber. Der Chemismus der Gährungsküpe beruht auf Bacterienwirkung und ist naturgemäss schwer regulirbar. Durch zu weit gehende Gährung kann vollkommene Zerstörung des Farbstoffes eintreten, und das Führen der Küpe erfordert ein besonderes Maass ron Aufmerksamkeit und Erfahrung.

Dagegen kennt man das Reductionsmittel der Hydrosulfitküpe auf das Genaueste. Fs ist eine Substanz von analytisch controlirbarem Wirkungswerth, welche klare Lösungen von Indigweissnatrium ohne dessen Zerfallsproducte liefert. In Folge dieser Vorzüge wird Baumwolle mit Hydrosulfit in grösstem Maassstabe geküpt. Für Wolle aber ist die Gährungsküpe unentbehrlich, und frägt man nach dem Grunde, so sind es nicht etwa die geringeren Betriebskosten, sondern es ist die Leichtigkeit, mit der hier tiefe und gleichmässige Durchfärbung der Faser erfolgt. Im Gegensatz dazu kommen in der Hydrosulfitküpe Störungen vor ${ }^{5}$ ).

Ferner theilte uns Herr Bũcheler damals Folgendes mit:

„Ovid in der ars amandi 3, 163 nennt nur „Deutsche Kräuter", mit welchen die (römische) Frau ihre grauen Haare färbe. Solche Färbemittel werden massenhaft erwähnt, zu Martial's Zeit (Ende des 1. Jahrh.) war die spuma Batava, sp. Chattiva, sp. Mattiaca - also ein in Hessen und Friesland, zwischen Wiesbaden und Arnhoim bereitetes, resp. dorther über den Rhein exportirtes halbflüssiges Product - für jenen Zweck berühmt, wie zu Plinius' Zeit (eine Generation früher) ein Sapo Gallicus, irgend ein Seifenpräparat. Von der isatis speciell als germanischem Färbemittel redet Niemand, wohl aber Plinius wie schon die Griechen allgemein von deren Färbekraft und dass die Wollfabrikanten sich ibrer bedienten. Über die kosmetischen Mittel, in denen das Alterthum sehr erfinderisch, und die betreffenden Färbstoffe finden Sie mehr bei Beckmann, Geschichte der Er findungen IV, p. 6 ff. Die Stellen aus den Alten giebt Marquardt, Privatalterthümer der Römer, p. 764 der 2. Auflage an. Der Ovidische Vers (um Christi Geburt) lantet:

Femina canitiem Germanis inficit herbis.

Deutsch waid weid stellt etymologisch die Wurzel von ioarıs dar, zum selben Namen gehört lat. vit-rum."

6) "Lose Wolle zeigt in der Hydrosulfitküpe zuweilen sogenannte "Spitzen“. Die Enden der einzelnen Wolllocken - die häufig noch etwas Wollfett enthalten, das besonders bei dem viel verwondeten überseeischen Material schwierig zu entfernen ist - bleiben heller und erscheinen hāufig sogar ganz ungefärbt. Dieser Übelstand kommt in der Gährungsküpe scltener vor. Stückwaare wird in der Hydrosulfitkūpe schwieriger durchgefärbt. Für dunkle Töne auf loser Wolle und Stückwaare findet aus den erwähnten Gründen die Hydrosulfit- 
Um ein Chinon zu einem Hydrochinon zu reduciren, greift man als Organiker nicht zu der Waidpflanze und ihren Mikroorganismen. Dass der Indigofärber das thun muss, trotz der fortwährend dabei drohenden Farbstoffverluste, ist ein Missstand. Aber eine Abhülfe kennt man einstweilen nicht; man steht also vor der Thatsache, dass es für einen der schönsten organischen Körper keine Anwendungsform giebt, die mit einem so veralteten Verfabren, wie es die Gährungsküpe ist, aufräumen könnte, obwohl über den Indigo Generationen von Chemikern gearbeitet haben.

Ein Weg des Fortschrittes dürfte hier in dem Studium derjenigen Umstände liegen, die der Einführung der Hydrosulfitküpe entgegenstehen, und dazu will die vorliegende Arbeit beitragen.

Die in ihr beschriebenen Versuche gründen sich auf folgende Betrachtung.

In der Hydrosulfitküpe verwendet man zur Reduction des Indigos Natriumhydrosulfit, $\mathrm{Na}_{2} \mathrm{~S}_{3} \mathrm{O}_{4}$, und zur Auflösung des entstandenen Indigweiss Natronlauge oder Kalk. Selbstverständlich werden die Mengen dieser Substanzen genau bemessen, und die Zusammensetzung der Küpenlösung wäre demgemäss eine recht einfache, wenn sie nicht in Folge des eigenthümlichen Verhaltens des Natriumhydrosulfits sich in uncontrolirbarer Weise änderte. Diese Substanz wird nämlich, wie Bernthsen ${ }^{7}$ ) gezeigt hat, durch Oxydation sauer, indem sie pyrosch wef ligsaures Salz giebt:

$$
\underset{\text { Natriumbydrosulfit }}{\mathrm{Na}_{2} \mathrm{~S}_{2} \mathrm{O}_{4}+\mathrm{O}}=\underset{\text { Natrinmpyrosalfit }}{\mathrm{Na}_{2} \mathrm{~S}_{2} \mathrm{O}_{5}}
$$

und letzteres absorbirt Alkali:

$$
\underset{\text { Natriumpyrohulfit }}{\mathrm{Na}_{2} \mathrm{~S}_{2} \mathrm{O}_{5}+2 \mathrm{NaOH}}=\underset{\text { Natriumanlft. }}{2 \mathrm{Na}_{2} \mathrm{SO}_{3}+\mathrm{H}_{2} \mathrm{O}}
$$

In Folge dessen sinkt durch Eindringen der Luft der Alkaligehalt der Küpe, während sich Natriumsulfit entsprechend anreichert. Ferner verändert sich das Reductionsmittel durch intramoleculare Oxydation, wobei es in Thiosulfat und Pyrosulfit zerfällt $t^{8}$ ). Da alle diese Reactionen stetig fortschreiten, so verschwinden allmählich Natronlauge und Natriumhydrosulfit aus der Flotte, und an ihre Stelle treten Natriumpyrosulfit, Natrium-

küpe nur beschränkte Verwendung und hier hat die Gährungsküpe in vollem Umfange das Feld behauptet." (Indigobuch B. A. S. F., S. 146, vergl. daselbst S. 125.)

7) Ann. Chem. Pharm. 208, 161 (1881). Vergl. ferner Bernthsen und Bazlen, Ber. Deutsch. Chem. Ges. 33, 130 (1900).

s) Bernthsen (a. a. O.) formulirt diesen Vorgang folgendermaassen:

$$
\underset{\text { Natriumbydrosulat }}{2 \mathrm{~S}_{2} \mathrm{O}_{4} \mathrm{Na}_{2}}=\underset{\text { Natriumthiosulfat }}{\mathrm{S}_{2} \mathrm{O}_{3} \mathrm{Na}_{2}}+\underset{\text { Natrlumpyrosalfit }}{\mathrm{S}_{2} \mathrm{O}_{5} \mathrm{Na}_{2}}
$$

thiosulfat, Natriumsulfit und schliesslich Natriumsulfat. Bei längerem Gebrauch der Küpe, um den es sich fast immer handelt, macht diese Umwandlung ihrer anfänglichen Beschaffenheit stetige Zugaben von Natronlauge und Natriumbydrosulfit erforderlich, da sonst Indigweiss oder Indigblau ausfallen würden, und darum ist es sehr bald unmöglich, die Mengen der anorganischen Bestandtheile in der Flotte auch nur schätzungsweise anzugeben ${ }^{9}$ ).

Zwar lassen sich die Schwankungen des Ätznatrongehaltes innerhalb gewisser Grenzen halten, da die Faser Alkali absorbirt, die Concentration desselben also auch bei längerem Färben und dem dadurch bedingten Zugeben von Natronlauge oder von Stammküpe nicht anzuwachsen braucht. Anders dagegen mit den durch Oxydation des Hydrosulfits entstehenden Salzen. Sie häufen sich in der Küpe ${ }^{10}$ ). Es dürfte schwer sein, sie quantitativ neben Indigweissnatrium zu bestimmen, und eine Methode zur Ermittlung der Alkaliconcentration in der Hydrosulfitküpe giebt es überhaupt nicht" ${ }^{11}$ ).

Man färbt also in der Hydrosulfitküpe bei Gegenwart unbekannter Alkali- und Salzmengen, und es erhebt sich die Frage, ob darin die Ursache der Schwierigkeiten liegt, die sich dem Erzielen gleichmässiger und tiefer Nuancen entgegenstellen.

Dass ein Übermaass ron Alkali das $\mathrm{Zu}$ standekommen tiefer Färbungen verhindert, ist qualitativ bekannt ${ }^{12}$ ). Dagegen ist der Alkalieinfluss quantitativ noch nicht verfolgt

9) Es ist für diese Betrachtungen gleichgültig, ob das Hydrosulfit vom Färber selbst ans Natriumbisulfit, Zinkstaub und Kalk oder nach dem D.R.P. 112483 (s. Bernthsen u. Bazlen a. a. O.) bereitet wird. In ersterem Fall gesellt sich zu den oben erwähnten Salzen meist noch Zinkoxydnatrium.

10) „Je länger auf der Küpo gefärbt wird, um so grösser wird ihr Gehalt an Salzen durch das fortgesetzto Zugeben von Stammküpe. Thr Eigengewicht steigt von $1 / 2^{\circ}$ Bé auf etwa $10^{\circ}$ Bé, und sie färbt in diesem Zustand etwas streifig." (Indigobuch B.A.S.F. S. 84.)

11) Man kann die Alkalität der Hydrosulfitkūpe in der Weise controlliren, dass man eine Probe oxydirt, filtrirt und titrirt. Selbstrerständlich wird dabei ein Theil des Alkalis durch das entstehende Pyrosulfit neutralisirt. Dis gefundenen Zahlen geben also nicht den Alkaligehalt der unoxydirten Küpo, sind aber immerhin zur Controle des Betriebes von Nutzen. Die Titration des Alkalis ohne vorausgegangene Oxydation des Hydrosulfits dürfte schon daran scheitern, dass letztere Substanz die Indicatoren entfärbt. Curcuma ist, wie wir gefunden haben, einige Zeit bestāndig. - Mit Versuchen zur Bestimmung der Alkalität unoxydirter Hydrosulfitküpen sind wir beschäftigt.

12) Siehe das Indigobuch B.A.S.F. S. 83, 149, 15̌1, 153. 
worden, und über eine etwaige Wirkung der Salze des Hydrosulfitansatzes weiss man überhaupt nichts. Man könnte höchstens aus einem Patent ron $\mathrm{Gutbier} \& \mathrm{Co}^{{ }^{13}}$ ) schliessen, dass geringe Mengen von Neutralsalzen in der Küpe ohne Belang sind. In Anbetracht der oben dargelegten Verhältnisse schien aber dennoch ein genaueres Studium der Salzwirkung angezeigt, und es sei vorgreifend erwähnt, dass dabei ganz andere Resultate erhalten wurden, als man nach der Gutbierschen Patentschrift hätte erwarten sollen.

Im Folgenden sind Versuche mitgetheilt, die zunächst zur Kenntniss der Wirkungsweise des Alkalis in der Küpe weiteres ${ }^{14}$ ) quantitatives Material bringen. Sodann wird die Frage erörtert: Welches ist der Einfluss von Salzen in der Indigoküpe?

\section{Arbeitsmethode.}

Der Hauptbestandtheil der Hydrosulfitkūpe ist Indigweissnatrium. Wir haben deshalb, um die erwähnten Punkte klarzulegen, versucht, möglichst neutrale Lösungen von Indigweissnatrium darzustellen, und dann die Änderung geprüft, welche ihr Färbevermögen durch den Zusatz von Natronlauge und von Salzen erfăhrt. Die Verănderlichkeit, welcher verdünnte Lösungen von Indigweiss in Alkali unterworfen sind, gestaltete dis Arbeit zu einer recht schwierigen, denn selbstverstandlich sinkt durch Luftzutritt der Gehalt an Indigweiss, indem sich Indigo ausscheidet, und zugleich wird die entsprechende Menge Alkali frei. Es wird später gezeigt werden, dass sehr geringfügige Ānderungen im Alkaligehalt von erbeblichem Einfluss auf das farberische Resultat sind. Beim Arbeiten mit den verhăltnissmåssig kleinen Laboratoriumsküpen musste also die Luft auf das sorgfältigste ausgeschlossen werden. Nach vielen erfolglosen Versuchen erwies sich schliesslich folgende Methode als die geeignete:

Sechs Liter destillirtes Wasser wurden in einem bis zum Halse gefullten Kolben zwei Stunden am Rückflusskǔhler heftig gekocht und so von Luft befreit. Eine frisch entkorkte Flasche, enthaltend ungefāhr $100 \mathrm{~g}$ 15-proc. Indigweisspaste B.A.S.F., wurde, an einer Schnur hăngend, in das noch heisse Wasser

13) D.R.P. No. 66687 (1891). Patentanspruch: "Verfahren der Küpenfārberei, darin bestehend, dass zur beschleunigten Aufnahme des Indigweiss durch die Faser und Erzielung eines tieferen Blaus der fertigen Küpe 5 Proc. Chlornatrium oder äquivalenter Mongen eines der folgenden Salze zugesetzt werden, nümlich" .... (folgen 13 Salze) . . . nund dass durch späteren Zusatz der betreffenden Salze das specifische Gewicht der Küpenflotte constant erhalten bleibt."

Die Beschrãnkang des Patentanspruches anf 5 Proc. Salz (N.B. bezogen auf den Cubikinhalt der Küpe, nicht etwa auf das Gewicht der Faser) beweist, dass geringere Salzmengen für gleichgültig gehalten werden.

$\left.{ }^{14}\right)$ Siehe unsere III. Mittheilung. hineingelassen, so dass die Öffnung nach oben zeigte und sich eine mit Natronlauge gefüllte Pipette unter dem Wasserspiegel in die Flasche einführen liess. Die Natronlauge war aus $\mathrm{Na}$ triumcarbonat und Kalk frisch bereitete Normallösung. Sie erwies sich bei der Titration als frei von Carbonat ${ }^{15}$ ).

Das Indigweiss wird vom heissen Wasser reichlich aufgenommen und geht durch Zufluss der Natronlauge vollends in Lösung, soweit die Alkalimenge das zulāsst. Letztere wurde so bemessen, dass auf 6 Liter Küpe ungefähr $2,5 \mathrm{~g}$ Ätznatron kamen, das Indigweiss also sich im Überschuss befand.

Nach Einbringung der Natronlauge wurde die Indigweissflasche mit der Schnur auf den Boden des Kolbens hinabgelassen und letzterer mit einem doppelt durchbohrten Kautschukstopfen verschlossen, in dem zwei Glasröhren staken. Durch sie strömte ein kräftiger Strom von sauerstofffreiem Stickstoff ${ }^{16}$ ) und bewirkte Durchmischung des Kolbeninhaltes. Der Stickstoff trat unter Wasserverschluss aus. Nachdem der Kolbeninhalt sich abgekūhlt hatte, wurden die auf den Glasröhren sitzenden Schläuche durch Quetschhãhne verschlossen und der Kolben vorsichtig umgekehrt, so dass eine nochmalige Durchmischung des Inhaltes stattfand. Nach vollkommenem Absitzen des Indigweissbodensatzes begannen die Fărbeversuche.

Es handelte sich bei ihnen darum, innerhalb jeder Serie von Ausfärbungen den Gehalt der Küpo an Indigweissnatrium constant zu halten, dagegen den Gehalt an Salzen und an Átznatron einzeln oder zugleich zu variiren. $\mathbf{Z u}$ dem Zweck wurden die Zusâtze von Salz und von Ātznatron in der stets gleich bleibenden Menge von $75 \mathrm{ccm}$ Wasser gelöst und in einem Mischcylinder mit Küpenflüssigkeit auf genau $1000 \mathrm{ccm}$ aufgefüllt. Vorher wurde der Mischeylinder mit Wasser gefüllt und dann das Wasser durch Stickstoff verdrāngt. Die Küpenflüssigkeit wurde jedesmal ans der 6-Liter-Küpe durch Stickstoff hinübergedrückt. Darauf erfolgte Schliessen und Umkehren des Mischeylinders. Der Verschluss bestand in einem zweifach durchbohrten Kautschukstopfen, in dem sich Glasröhren befanden, die ihrerseits durch Schlåuche und Quetschhähne verschlossen waren. Nach dem Durchmischen wurden $200 \mathrm{ccm}$ Flüssigkeit zur Analyse entnommen unter Verbinden des einen Glasrohres mit der Stickstoffquelle und Hinüberdrücken der Lösung in einen Messkolben, an dessen Boden sich etwas Benzol befand. Die übrigen im Cylinder befindlichen $800 \mathrm{ccm}^{17}$ ) wurden nun zum Färben von $15 \mathrm{~g}$ loser, gewaschener Wolle verwandt. Letztere durfte aber

15) Siehe Petersen, Zeitschr. angew. Chemie 1900 , S. 688.

16) Bereitet nach der Methode von Tichborne (siehe L. Braun, Zeitschr. phys. Chem. 83, 725, 1900). Leuchtgas ist zwar weit boquemer zugänglich, aber weniger sicher, da es so leicht herausdiffundirt.

17) In einigen Fällen fanden Abweichungen von diesen Zahlen statt, die Arbeitsmethode war aber dann im Princip dieselbe. 
nicht in den Cylinder eingeführt werden, da dabei Sauerstoff hinzugetreten wäre. Die Wolle wurde deshalb in einem zweiten Cylinder, der wie der erste verschlossen war, gleichmässig vertheilt; sodann wurde die Luft zuerst durch Leuchtgas, dann vollends durch Stickstoff aus dem zweiten Cylinder verdrängt und jetzt gelangten - wiederum durch den Druck einer mit Stickstoff gefüllten Flasche und nach Verbindung der betreffenden Glasröhren - die gesammten $800 \mathrm{ccm}$ des Mischeylinders in den Färbecylinder.

Während aller dieser Operationen standen die 3 Gefăsse, 6-Liter-Küpe, Mischcylinder und Färbecylinder, in einer grossen Wanne voll Wasser, dessen Temperatur durch einen Thermostaten auf $50^{\circ}$ gehalten wurde.

Die Küpenlösung in der 6-Liter-Küpe war stets hellgelb und vollkommen klar. Das Glasrohr, aus welchem der Ausfluss stattfand, tauchte nur so tief ein, dass ron dem aus Indigweiss bestehenden Bodensatz nichts mitgerissen werden konnte.

Vor dem Färben mit Alkali- oder Salzzusatz fand bei jeder Versuchsreihe eine Färbung mit salzfreier Küpe statt. Dabei wurden $75 \mathrm{ccm}$ destillirtes Wasser, also dieselbe Menge, die bei den Parallelversuchen zur Lösung der Zusätze diente, im Mischcylinder mit Küpenlösung zu- enthaltende Indigoküpen nach der Oxydation gekocht werden müssen, anderenfalls läuft der Indigo durchs Filter.

Sollte nach dem Färben der Alkali- und Salzgehalt der Küpe bestimmt werden, so wurden die Filtrate mit den Waschwässern vereinigt auf 300 ccm aufgefült und dann in $100 \mathrm{ccm}$ nach dem Ansäuern mit Salpetersãure das Kochsalz ${ }^{18}$ ) nach Volhard titrirt, in $200 \mathrm{ccm}$ nach dem Ansäuern mit Salzsäure und Eindampfen das Chlornatrium gewogen. Die Differenz ergab den Ätznatrongehalt. In den Küpen, die kein Kochsalz enthielten, wurde das Alkali als Sulfat bestimmt. Titrationen des Alkalis erwiesen sich wegen der grossen Verdünnung als für den vorliegenden $Z$ weck nicht hinreichend genau.

Ausser reinen Indigweissnatriumlósungen wurden auch hydrosulfithaltige, nach der üblichen Methode dargestellte, zur Untersuchung verwandt. Die Versuchsanordnung blieb in solchen Fãllen dieselbe. Die Alkalität der Küpe konnte aber dann aus den oben mitgetheilten Gründen nicht bestimmt werden.

\section{Wirkungsweise des Alkalis in der Wollküpe.}

Der Einfluss von Natronlauge auf das Küpen der Wolle ergiebt sich aus Tabelle $I$.

Tabelle $I$.

\begin{tabular}{|c|c|c|c|c|c|c|c|c|c|c|}
\hline \multirow{3}{*}{$\begin{array}{c}\text { No. } \\
\text { des } \\
\text { Ver- } \\
\text { suches }\end{array}$} & \multirow{3}{*}{$\begin{array}{c}\begin{array}{c}\text { Woll- } \\
\text { gewicht }\end{array} \\
\text { g }\end{array}$} & \multirow{3}{*}{$\begin{array}{c}\text { Vol. } \\
\text { der } \\
\text { Farbo- } \\
\text { ktpo } \\
\text { ccm }\end{array}$} & \multicolumn{4}{|c|}{ In $100 \mathrm{ccm} \mathrm{Küpe}{ }^{19}$ ) befinden sich: } & \multirow{3}{*}{$\begin{array}{l}\text { Ansuehen } \\
\text { der } \\
\text { gorkrbten } \\
\text { Wolle }\end{array}$} & \multirow{3}{*}{$\begin{array}{c}\text { MIt Fiseculg } \\
\text { extrahirto } \\
\text { Wollo } \\
\text { g }\end{array}$} & \multirow{3}{*}{$\begin{array}{c}\text { Abge- } \\
\text { zogener } \\
\text { Indigo } \\
\mathrm{g}\end{array}$} & \multirow{3}{*}{$\begin{array}{c}\text { Indigo } \\
\text { berechnot } \\
\text { in Proa. } \\
\text { des } \\
\text { Woll. } \\
\text { gevichtes }\end{array}$} \\
\hline & & & \multicolumn{2}{|c|}{ Indigweiss } & \multicolumn{2}{|c|}{ Ätznatron } & & & & \\
\hline & & & $\begin{array}{c}\text { vor dem } \\
\text { Furben } \\
\text { g }\end{array}$ & $\begin{array}{c}\text { anoh dem } \\
\text { Fidrbon } \\
\text { E }\end{array}$ & $\begin{array}{c}\text { vor dem } \\
\text { Furben } \\
\text { g }\end{array}$ & $\begin{array}{c}\text { anch dem } \\
\text { Frarben } \\
\text { B }\end{array}$ & & & & \\
\hline $\begin{array}{l}1 \\
2\end{array}$ & $\begin{array}{l}30,0 \\
30,0\end{array}$ & $\begin{array}{l}1800 \\
1800\end{array}$ & $\begin{array}{l}0,1324 \\
0,1351\end{array}$ & & $\begin{array}{l}\left.0,0187^{19}\right) \\
\left.0,1707^{19}\right)\end{array}$ & & $\begin{array}{c}\text { mittelblau } \\
\text { hellblau }\end{array}$ & $\begin{array}{l}14,965 \\
16,402\end{array}$ & $\begin{array}{l}0,2247 \\
0,1087\end{array}$ & $\begin{array}{l}1,5 \\
0,66\end{array}$ \\
\hline $\begin{array}{l}3 \\
4\end{array}$ & $\begin{array}{l}15,0 \\
15,1\end{array}$ & $\begin{array}{l}800 \\
800\end{array}$ & $\begin{array}{l}0,0554 \\
0,0521\end{array}$ & $\begin{array}{l}0,0432 \\
0,0450\end{array}$ & $\begin{array}{l}0,0337 \\
0,1290\end{array}$ & $\begin{array}{l}0,0304 \\
0,1179\end{array}$ & $\begin{array}{c}\text { hellblau } \\
\text { sebr blass } \\
\text { blau }\end{array}$ & $\begin{array}{l}14,678 \\
15,060\end{array}$ & $\begin{array}{l}0,0270 \\
0,0581\end{array}$ & \\
\hline
\end{tabular}

sammengebracht. Der Indigweissgehalt blieb also bei den zu vergleichenden Fărbungen derselbe. Nach beendigtem Färben wurden $200 \mathrm{ccm}$ der Küpe aus dem Fãrbecylinder behufs Analyse durch Blasen an dem einen in den Cylinder führenden Glasrohr in einen Messkolben gedrūckt. Da der Anfangsgehalt der Küpe analysirt worden war, so ergab sich daraus und aus der zweiten Analyse der Grad der Erschópfung der Küpe. Der Rest der Küpe gelangte auf die gleiche Weise zum Ausfluss, so dass die Wolle allmählich von oben her Luftzufuhr empfing. Die Wolle wurde dann dem Gefäss entnommen und nach halbstündigem Vergrünen in einer bei jedem Versuche gleichbleibenden Menge Wasser gewaschen. Die an der luft getrockneto Faser wurde zur Indigobestimmung mit Eisessig extrahirt. Die zu vergleichenden Färbeproben kamen rasch hintereinander, also bei gloicher Luftfeuchtigkeit zur Wăgung, da der Wassergehalt der Wolle mit der Lufteuchtigkeit schwankt.

Betreffs der Analyse der Küpenlösungen ist zu erwăhnen, dass sehr verdünnte, kein Salz
Die Zeit des Färbens betrug 30 Minuten, die Temperatur 50 $0^{\circ}$ Die Bestimmung der

18) Die meisten Versuche wurden aus einem später anzuführenden Grunde mit Chlornatrium angestellt.

19) Ansatz der Stammküpen: für Versuch 1 und 2 ca. $25 \mathrm{~g}$ Indigweiss B. A. S. F. (in Form ron Paste), $75 \mathrm{ccm}$ Natriumhydrosulfitlösung (bereitet nach Indigobuch B.A.S.F. S. 76), 17,5 cem Natronlange $25^{\circ}$ Bé. Die Verdünnung bei Ansatz der Färbekūpe wurde so gewählt, dass die Menge des Alkalizusatzes $0,0187 \mathrm{~g}$ in $100 \mathrm{ccm}$ betrug. Der wirkliche Alkaligehalt entspricht dem nicht, da dieser aus den oben erwähnten Gründen in einer Hydrosulfitküpe nicht bestimmbar ist.

Küpe 3 und 4 enthielten kein'Hydrosulfit. Die aufgeführte Ätznatronmenge entspricht also dem wirklichen Gehalt der Küpe, wie er nach deren Oxydation ermittolt wurde. Vor der Oxydation war ein Theil davon an Indigweiss gebunden. Der Gohalt an freiem Alkali in der unoxydirten Küpe ergiebt sich aus der nach der Oxydation gefundenen Zahl abzüglich der dem Indigweiss äquivalenten Menge. Der Überschuss berechnet sich für Küpe 3 $\mathrm{zu} 0,0174 \mathrm{~g}$ in $100 \mathrm{ccm}$. 
Indigweissmengen vor dem Färben zeigt bei je zwei zu vergleichenden Küpen denselben Gehalt. Die Abweichungen liegen innerhalb derGrenzen der zulässigen analytischen Fehler.

Durch Zugabe ron Natronlauge zu Küpe 1 entstand 2, ebenso 4 aus 3. Das Resultat zeigte sich unzweideutig bei der Betrachtung der Ausfärbungen. Die Tiefe der Nuance hatte in beiden Fällen durch Alkalizusatz erheblich abgenommen.

Zur zahlenmässigen Belegung dieses Ergebnisses wurde die geküpte Wolle mit Eisessig extrahirt. Dabei ergaben aber nur Wollproben 1 und 2 hinreichend grosse Mengen, um einen Schluss zuzulassen, bei dem die Fehler der Eisessigmethode ${ }^{24}$ ) nicht zu sehr ins Gewicht fielen. Gestützt auf diese Analysen kann man sagen:

Bei einem Ätznatronzusatz von $\left.0,17 \mathrm{~g}^{21}\right)$ auf $100 \mathrm{ccm}$ Hydrosulfitk üpe nahm Wolle in diesem besonderen Falle nur rund die $\mathrm{Halfte}{ }^{23}$ ) derjenigen Indigweissmenge auf, die sie einer
Küpe ron $0,018 \mathrm{~g}$ Alkaliüberschuss in $100 \mathrm{ccm}$ entzieht. Bei einem früheren Versuch ${ }^{23}$ ) hatte sich ergeben, dass der gleiche Fffect beim Küpen von Baumwolle erst durch Zusatz von $0,45 \mathrm{~g}$ Ätznatron zu $100 \mathrm{ccm}$ neutraler Indigweisscalciumlösung erreicht wird. Die Alkaliwirkung tritt also in der Wollküpe stärker hervor als in der Baumwollküpe. Das war qualitatir bereits bekannt $\left.{ }^{2+}\right)$. Ein quantitatives Verhältniss ergiebt sich aus den mitgetheilten Zahlen; indessen bedeuten diese keineswegs Constante. Es wird sich weiter unten ${ }^{25}$ ) zeigen, dass die Alkaliwirkung in der Hydrosulfitküpe aus Gründen, die man bisher nicht kannte, eine durchaus schwankende ist.

III. Vorversuche über die Wirkungsweise ron Salzen in der Küpe.

Die in Tabelle II niedergelegten Versuche sollten darthun, ob Salze überhaupt in der Küpe wirksam sein können. Das Ergebniss war ein positives.

Tabelle $I I$.

\begin{tabular}{|c|c|c|c|c|c|c|c|c|c|c|}
\hline \multirow{3}{*}{$\begin{array}{l}\text { No. } \\
\text { des } \\
\text { Ver- } \\
\text { suches }\end{array}$} & \multirow{3}{*}{$\begin{array}{l}\text { Faner- } \\
\text { atoff }\end{array}$} & \multirow{3}{*}{$\begin{array}{c}\text { Salzzusatz } \\
\text { auf } \\
100 \mathrm{cem} \\
\text { Kupe } \\
\text { g }\end{array}$} & \multicolumn{4}{|c|}{$\begin{array}{l}\text { In } 100 \mathrm{ccm} \text { einer Kupe von } 800 \mathrm{ccm} \\
\text { Gesammtinhalt waren }\end{array}$} & \multirow{3}{*}{$\begin{array}{l}\text { Aussehed } \\
\text { der } \\
\text { gefurbten } \\
\text { Faser }\end{array}$} & \multirow{3}{*}{$\begin{array}{c}\text { Mit } \\
\text { Eloessig } \\
\text { extrab. } \\
\text { Fager } \\
\text { g }\end{array}$} & \multirow{3}{*}{$\begin{array}{c}\text { Abge- } \\
\text { zogener } \\
\text { Indigo } \\
\text { F }\end{array}$} & \multirow{3}{*}{$\begin{array}{c}\text { Indigo } \\
\text { ber. in } \\
\text { Proc. } \\
\text { des } \\
\text { Faser- } \\
\text { etoffes }\end{array}$} \\
\hline & & & \multicolumn{2}{|l|}{ Indigwelsy } & \multicolumn{2}{|c|}{ Natriumhydrozyd } & & & & \\
\hline & & & $\begin{array}{l}\text { vor } \\
\text { dem Furben } \\
\text { B }\end{array}$ & $\begin{array}{c}\text { nach } \\
\text { d.Farb. } \\
\text { R }\end{array}$ & \begin{tabular}{|c|} 
vor \\
d. Fyrb. \\
g
\end{tabular} & $\begin{array}{c}\text { nach } \\
\text { d. Färb. } \\
\text { g }\end{array}$ & & & & \\
\hline $\left.5^{96}\right)$ & $\begin{array}{c}15,50 \\
\text { Baumwolle }\end{array}$ & - & $\begin{array}{c}\text { nicht } \\
\text { analysirt }\end{array}$ & & \multirow{2}{*}{\multicolumn{2}{|c|}{$\begin{array}{c}\text { nicht } \\
\text { analysirt }\end{array}$}} & hellblau & 14,145 & 0,2505 & 1,77 \\
\hline 6 & $\begin{array}{c}15,5 \\
\text { Baumwolle }\end{array}$ & $\begin{array}{c}\text { 6,2 } \\
\text { Glaubersalz }\end{array}$ & $\begin{array}{l}\text { nicht analysirt, } \\
\text { identisch mit } \mathbf{5}\end{array}$ & $\left\{\begin{array}{l}1,089 \\
1,092\end{array}\right.$ & & & mittelblau & 15,184 & 0,3316 & 2,10 \\
\hline $\left.7^{27}\right)$ & 15,5 Wolle & 一 & 0,0542 & nicht & 0,061 & 0,051 & $\begin{array}{l}\text { sehr helles } \\
\text { Blau }\end{array}$ & 15,484 & 0,0511 & 0,33 \\
\hline 8 & 15,5 Wolle & $\begin{array}{l}\text { 1,0 Koch- } \\
\text { salz }\end{array}$ & 0,0510 & $\begin{array}{l}\text { ana- } \\
\text { lysirt }\end{array}$ & 0,061 & - & $\begin{array}{c}\text { wesentlich } \\
\text { dunkler }\end{array}$ & 15,350 & 0,1120 & 0,73 \\
\hline
\end{tabular}

20) Uber die Fobler der Fisessigmethodo bein Analysiren geküpter Wolle haben wir unlängst berichtet (s. d. Zeitschr. 1902, S. 557). Wie unzuverlässig die Methode ist, zeigte Versuch 3. Er ergab nāmlich für den abgezogenen Indigo einen viel zu geringen Werth, der mit dem Aussehen der Färbeproben in offenbarem Widerspruch stand. Da sehr sorgfältig gearbeitet wurde, so glauben wir nicht, dass ein analytisches Versehen vorliegt. Dagegen musste natürlich bei so geringen Mengen Farbstoff, wie sie die Wolle von Versuch 3 enthielt, die Ungenauigkeit der Methode schwer ins Gewicht fallen, und wahrscheinlich rührt daher die Abweichung von dem zu erwartenden Resultat.

21) Ein Zusatz von dieser Höhe liegt ausserhalb des Bereiches des technisch Zulässigen, da bei einer derartigen Alkaliconcentration die Wolle angegriffen wird. Bei obigen Versuchen wurden diese Verhältnisse gewählt, um möglichst grosse und mit Sicherheit analytisch feststellbare Unterschiede zu erhalten.

22) Der von der Wolle abgezogene lndigo wurde nach der Methode B.A.S.F. (Indigobuch S. 28) sulfurirt und titrirt. Es ergab sich bei Versuch 1 ein Reingehalt von 88,6 und bei Versuch 2 ein solcher von 89,5 Proc. Das Vergleichsresultat wird dadurch nicht geändert.
Aussehen und Analysen der gefärbten Fasermaterialien, ferner bei der Baumwollküpe die grössere Erschöpfung von 6, die durch Doppelanalysen belegt ist, alles das beweist, dass Wolle sowohl, wie Baumwolle sich bei Gegenwart von Neutralsalzen tiefer anfärben können als ohne dieselben.

Die Menge des zur Wollküpe (8) gegebenen Salzes betrug nur 1 Proc. der Flotte, also $1 / 5$ derjenigen Menge, die Gutbier in der citirten Patentschrift als wirksam angiebt. $\mathrm{Da}$ beim Hydrosulfitansatz mit derartigen Salzconcentrationen gerechnet werden muss,

23) Siehe unsere Mittheilung in dieser Zeitschr. 1900, S. 414, Verauch 5.

21) Indigobuch B.A.S.F. S. 42.

$25)$ Siehe Anm. 48

26) Zinkstaub-Ätznatronkūpe. Dauer des Färbeus bei 5 und 617 Stunden, Temperatur ungefăhr $17^{\circ}$.

27) Indigweissnatriumküpe ohne Hydrosulfit. Dauer des Fürbens bei 7 und 830 Minuten, Temperatur $50^{\circ}$. 
so folgt, dass hier das Natriumbydrosulfit und dic aus ihm entstehenden Salze die Intensitït der Färbung beeinflussen können, falls sie gerade so wirken wie Chlornatrium. Wir werden später sehen, dass das der Fall ist.

Bevor zu einem weiteren Studium dieser Erscheinung geschritten wurde, war ein Punkt zu erledigen, dessen Nichtbeachtung leicht den Werth der ganzen mühevollen Arbeit hätte beeinträchtigen können.

Einer Untersuchung von Knecht zufolge nimmt nämlich Wolle beim Kochen mit verdünnten Chlornatriumlösungen merkbare Mengen des Salzes auf ${ }^{23}$ ), und auf Grund dessen könnte man sich die Salzwirkung in der Küpe folgendermaassen denken: In und auf der Wollfaser bildet sich eine stärkere Salzconcentration als in der Flotte, und dadurch findet eine Aussalzung des Indigweissnatriums auf der Wolle und ein tieferes Anfärben derselben statt. Um einen derartigen Zusammenhang zwischen Salz- und Farbstoffaufnahme darzuthun, hätten bei weiteren Versuchen die beim Färben adsorbirten Salzmengen bestimmt werden müssen; es war deshalb angezeigt, zuvor festzustellen, ob eine Salzaufnahme, wie Knecht sie in der Siedehitze fand, bei der Temperatur der Wollküpe $\left(50^{\prime \prime}\right)$ ebenfalls stattfindet.

\section{Verhalten der Wolle gegen Chlornatrium.}

Die Versuche bestanden darin, dass gewaschene lose Wolle ${ }^{29}$ ) mit warmer Kochsalzlösung, theils bei Gegenwart von Natronlauge, behandelt wurde, und dass vor- und nachher eine Bestimmung der Salzconcentration stattfand.

Das Chlornatrium war hier wie bei allen übrigen in dieser Arbeit mitgetheilten Versuchen chemisch reine Substanz. Sie wurde gewonnen durch Einleiten von Salzsäuregas in eine concentrirte Lösung von Speisesalz und Waschen und Trocknen des ausgeschiedenen Krystallbreis.

Die Wolle enthielt die für gereinigte lose Wolle normale Menge anorganischer Substanz: $7,6706 \mathrm{~g}$ verascht gaben $0,0615 \mathrm{~g}$ Rückstand $=0,8$ Proc. Bei einstündiger Behandlung mit siedendem Wasser gaben $15 \mathrm{~g}$ Wolle Spuren eines gelblichen Körpers an das Wasser ab, der mit Silberaitrat bei Gegenwart von Salpetersäure einen braunen flockigen Niederschlag erzeugte. Es war aber sehr wenig und wurde bei den Chlorsilberfällungen nach Behandeln der Wolle mit Salzlösungen vernachlässigt.

Die Versuche gaben folgende Resultate:

Tabelle III.

\begin{tabular}{|c|c|c|c|c|c|c|c|}
\hline \multirow{2}{*}{ 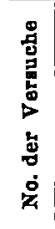 } & \multirow{2}{*}{$\begin{array}{c}\text { Gew, d. } \\
\text { Wolle }\end{array}$} & \multirow{2}{*}{ 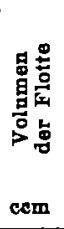 } & \multirow{2}{*}{ 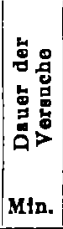 } & \multirow{2}{*}{ 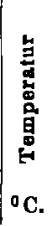 } & \multicolumn{2}{|c|}{$\begin{array}{l}\text { Chlornatzium- } \\
\text { gehalt }{ }^{22} \text { ) von } \\
100 \mathrm{ccm} \text { d. Flotte }\end{array}$} & \multirow{2}{*}{ 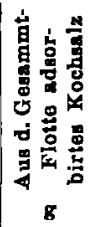 } \\
\hline & & & & & $\begin{array}{c}\text { Vor Ein- } \\
\text { bringen } \\
\text { d. Wolle } \\
\text { g }\end{array}$ & $\begin{array}{c}\text { Nach Ein } \\
\text { bringen } \\
\text { d. Wollo } \\
g\end{array}$ & \\
\hline 9 & & & 60 & 60 & & & 0,015 \\
\hline 10 & $15.338^{30}$ & 900 & 30 & 60 & 0,20 & & \\
\hline 11 & $15,383^{31}$ & 900 & 60 & 60 & 0,2039 & 0,2036 & 0,003 \\
\hline
\end{tabular}

Die in der Tabelle verzeichneten scheinbaren Differenzen der Kochsalzconcentrationen vor und nach der Immersion der Wolle sind äusserst gering und verschwinden so gut wie ganz, wenn die Wolle gründlich gereinigt wird (s. die Fussnoten 30 und 31). Es wird also unter den hier gewählten Versuchsbedingungen kein Chloraatrium aufgenommen.

Um den Verhältnissen in der Küpe Rechnung zu tragen, wurde weiter untersucht, wie sich Wolle gegen Kochsalz verhält, wenn ausser diesem auch noch Natronlauge zugegen ist. Die Resultate sind folgende:

Tabelle $7 \mathrm{~V}$.

\begin{tabular}{|c|c|c|c|c|c|c|c|c|}
\hline \multirow[b]{2}{*}{$\begin{array}{l}\text { No. der } \\
\text { Vers. }\end{array}$} & \multirow[b]{2}{*}{ Wollgewicht } & \multirow[b]{2}{*}{$\begin{array}{l}\text { Yol, d, } \\
\text { Flotte } \\
\text { cam }\end{array}$} & \multirow[b]{2}{*}{$\begin{array}{l}\text { Daner } \\
\text { d. Vers. } \\
\text { Min. }\end{array}$} & \multirow[b]{2}{*}{$\begin{array}{c}\text { Temp. } \\
{ }^{\circ} \mathrm{C} .\end{array}$} & \multicolumn{3}{|c|}{$100 \mathrm{ccm}$ der Flotte enthalten: } & \multirow[b]{2}{*}{$\begin{array}{c}\text { Corrlgirte } \\
\text { Zablen } \\
\text { (B. unten) } \\
\text { B }\end{array}$} \\
\hline & & & & & $\begin{array}{c}\text { Na OH ror dem } \\
\text { Finbringen der } \\
\text { Wolle } \\
\text { B }\end{array}$ & $\begin{array}{c}\text { Na Cl vor dem } \\
\text { Einbringen der } \\
\text { Wolle } \\
\text { B }\end{array}$ & $\begin{array}{c}\text { Na Cl nach dem } \\
\text { Elnbringen der } \\
\text { Wolle } \\
\text { (uncorrigirt) } \\
\text { B }\end{array}$ & \\
\hline $\begin{array}{l}12 \\
13 \\
14 \\
15\end{array}$ & $\begin{array}{r}15,099 \\
15,087 \\
15,312 \\
5,019\end{array}$ & $\begin{array}{l}900 \\
900 \\
900 \\
500\end{array}$ & $\begin{array}{l}30 \\
30 \\
30 \\
60\end{array}$ & $\begin{array}{l}60 \\
60 \\
60 \\
60\end{array}$ & $\begin{array}{l}0,0432 \\
0,0432 \\
0,0432 \\
0,0710\end{array}$ & $\begin{array}{l}0,2032 \\
0,3068 \\
0,2039 \\
0,1952\end{array}$ & $\begin{array}{l}0,2067 \\
0,3088 \\
0,2046 \\
0,1953\end{array}$ & $\begin{array}{l}0,2045 \\
0,3066 \\
0,2024 \\
0,1931\end{array}$ \\
\hline
\end{tabular}

2s) Chem.-Ztg. 12, 1171, 1888. Die aufgenommenen Mengen sind:

bei $0,048 \mathrm{NaCl}$ in $100 \mathrm{ccm} 0,29$ Proc. des Wollgew.

- $\mathbf{0 , 4 8 5}$

0,23

In Löwentha I's Handbuch der Färberei, II. Aufl. S. 138, heisst es, dass Wolle beim Kochen nur Spuren Chlornatrium aufnimmt. Diese "Spuren" betragen ungefähr soviel wie die Farbstoffmengen bei einer mittelstarken Küpenfärbung, ein Einfluss des adsorbirten Salzes beim Küpenprocess konnte also bei vorliegender Arbeit nicht als ausgeschlossen gelten.

29) Von der Badischen Anilin-u. SodaFabrik freundlichst zur Verfügung gestellt.

30) Die Wolle wurde vor dem Versuch im Soxhlet 3 Stunden mit Äther extrahirt.

31) Die Wolle wurde vor dem Versuch 2 Stunden mit Wasser ausgekocht.

32) Als Chlorsilber gefällt. 
Bei dieser Tabelle fällt es auf, dass die Zahlen für das Chlornatrium nach der Immersion der Wolle höher sind als vorher. Da beim Behandeln von Wolle mit Natronlauge Wollsubstanz unter Bildung von Lanuginsäure $^{33}$ ) in Lösung geht, so war zu vermuthen, dass bei der Fällung mit Silbernitrat neben dem Chlorsilber auch lanuginsaures Silber ausfiel, und dass darum die Resultate zu hoch erschienen. Zur Prüfung dessen wurde Wolle mit Natronlauge erwärmt, dann die von der Wolle abfiltrirte Lösung mit Salpetersäure angesäuert und mit Silbernitrat versetzt. Es fiel ein brauner Niederschlag aus, der auf einem Filter von bekanntem Gewicht bei $100^{\circ}$ getrocknet wurde. Es ergab sich Folgendes:
Salzes auf die Tiefe der Küpenfürbung kann also nicht darauf beruhen, dass die Concentration des Salzes und seine den Farbstoff ausfällende Wirkung in oder auf der Faser grösser istals in den übrigen Theilen der Küpe.

\section{Färbeversuche unter Zusatz von} Chlornatrium.

Im Anschluss an die vorigen Versuche wurde auch bei den folgenden Chlornatrium verwandt. Die Einzelheiten der Arbeitsmethode sind bereits in Abschnitt I beschrieben. Die Resultate ergeben sich aus Tabelle VI.

In Tabelle VI befinden sich zwei Serien von Versuchen. Die erste $(20-35)$ betrifft Lösungen von Indigweiss in Natronlauge, die

Tabelle $V$.

\begin{tabular}{|c|c|c|c|c|c|c|c|c|}
\hline $\begin{array}{l}\text { No. der } \\
\text { Verauche }\end{array}$ & $\begin{array}{c}\text { Woll- } \\
\text { gewicht } \\
\text { E }\end{array}$ & $\begin{array}{l}\text { Volumen } \\
\text { der Flotte } \\
\text { ccm }\end{array}$ & $\begin{array}{c}\text { Darin } \\
\text { Ätmatron } \\
\mathbf{B}\end{array}$ & $\begin{array}{l}\text { Daver der } \\
\text { Veraucho } \\
\text { MIn. }\end{array}$ & $\begin{array}{c}\text { Temp. } \\
{ }^{\circ} \mathrm{C} .\end{array}$ & $\begin{array}{c}\text { Nach dem } \\
\text { Vercheh mit } \\
\mathrm{Ag} \mathrm{NO}_{3} \\
\text { gefwllt } \\
\text { cem }\end{array}$ & $\begin{array}{c}\text { Darin } \\
\text { Niederschleg } \\
\mathbf{g}\end{array}$ & $\begin{array}{c}\text { Nlederschlıg } \\
\text { ber. auf } 100 \mathrm{ccm} \\
\mathrm{g}\end{array}$ \\
\hline $\begin{array}{l}16 \\
17 \\
18 \\
19\end{array}$ & $\begin{array}{l}14,93 \\
15,14 \\
15,19 \\
14,99\end{array}$ & $\begin{array}{r}1000 \\
1000 \\
1000 \\
900\end{array}$ & $\begin{array}{l}0,432 \\
0,432 \\
0,432 \\
0,432\end{array}$ & $\begin{array}{l}30 \\
30 \\
30 \\
30\end{array}$ & $\begin{array}{l}60 \\
60 \\
60 \\
60\end{array}$ & $\begin{array}{l}200 \\
400 \\
800 \\
800\end{array}$ & $\begin{array}{l}0,0106 \\
0,0204 \\
0,0455 \\
0,0459\end{array}$ & $\begin{array}{l}0,0053 \\
0,0051 \\
0,0057 \\
0,0057\end{array}$ \\
\hline
\end{tabular}

Es geht also eine Menge Wollsubstanz in Lösung, die bei einer gewissen Natronlaugeconcentration eine ganz constant bleibende Menge des Silberniederschlages liefert. Derselbe beträgt im Mittel $0,0054 \mathrm{~g}$ für $100 \mathrm{ccm}$ und ist ron den Chlorsilberzahlen, die der Tabelle IV zu Grunde liegen, abzuziehen. Man erhält dann die in der letzten Columne von Tabelle IV verzeichneten ${ }_{n}$ corrigirten Zahlen "34). Ihre Abweichungen von den Kochsalzconcentrationen vor Einbringung der Wolle sind sebr gering und rühren von den unvermeidlichen analytischen Ungenauigkeiten her.

$\mathrm{Da}$ in der Patentschrift von Gutbier die Hypothese aufgestellt wird, dass Indigweiss mit Neutralsalzen Doppelsalze bildet, welche auf die Faser ziehen, so haben wir schliesslich noch geprüft, ob Wolle bei Gegenwart von Indigweissnatrium entsprechende Mengen Chlornatrium aufnimmt. Auch das ist nicht der $\mathrm{Fall}^{35}$ ). Man kann also sagen:

Bei den für die Indigoküpe geltenden Bedingungen wird Chlornatrium seiner Lösung durch Wolle auch nicht in Spuren entzogen. Der Einfluss des

3a) Kne cht, Ber. Deutsch. chem. Ges. 22, 1120.

34) Der Versuch, die Correctur so auszuführen, dass vor Fällung des Salzes die vom Alkali aufgelöste Wollsubstanz mit Åther ausgeschüttelt wurde, gelang nicht. Ebensowenig kann man sie quantitativ mit Kupferacetat ausfällen.

a5) Die Resultate finden sich in Anm. 37 zu Tabelle VI. zweite (36-42) solche bei gleichzeitiger Anwesenheit von Natriumhydrosulfit. Beide Serien sind nach steigenden Salzzusätzen geordnet.

In jedem Fall betrug die Dauer des Färbens 30 Minuten, die Temperatur $50^{\circ}$. Vergrünen und Waschen geschah stets in vollkommen gleichmässiger Weise.

Durch die in Abschnitt I beschriebenen Cautelen wurde es erreicht, dass der Anfangsgehalt an Indigweiss bei den zu vergleichenden Küpen stets derselbe war. Das kornmt in den Analysen der fünften Verticalspalte zum Ausdruck.

Die Wirkung des Salzes zeigte sich an dem Aussehen der gefärbten Wolle nach dem Waschen und Trocknen (s. die letzte Verticalspalte). Bei $0,073 \mathrm{~g}$ Chlornatrium in $100 \mathrm{ccm}$ Küpe findet schon eine bemerkbare Vertiefung der Nuance statt (20 und 21). Dieselbe nimmt mit steigender Salzconcentration zu und liess sich bei 1,8 Proc. Kochsalz auf etwa das Doppelte der ursprünglichen Farbstärke schätzen (Versuche 22-27).

Wird die Wirkung des Salzes durch die Stärke der Natronlauge beeinflusst? Diese Frage findet in Versuchen 28-42 ihre Beantwortung. Die betreffenden Küpen entsprechen in den Salzmengen den vorhergehenden. Der Alkaligehalt dagegen ist bei 28-31 rund der dreifache, bei 32-35 etwa der zehnfache wie in 20-27. Das Resultat ist folgendes: Bei mässiger Alkalisteigerung 
(Versuch 28-31) vertieft sich durch Salzzusatz die Farbnuance etwa in demselben Maasse wie vorher, bei weiterem Alkalizusatz dagegen $(32-35)$ schwächt sich die Salzwirkung merkwürdiger Weise ab. Thatsächlich waren Ausfärbungen 33 und 34 nur sehr wenig von 32 verschieden, obgleich sie bei Gegenwart von Salzmengen entstanden waren, die sich bei $23,25,26,29$ und 30 von erheblichem Einfluss gezeigt hatten. Nur bei 35 war eine deutliche Änderung gegen die Anfangsfärbung zu bemerken. auf 0,0057 Proc. beschränkt werden, was beim Auflösen von Indigweiss in sehr verdünnter Natronlauge nicht gelungen war. Dabei fiel es auf, dass 0,073 und 1,8 Proc. Salz bei 38 und 39 keine so grosse Vertiefung der Nuance mit sich brachten als bei 41 und 42 .

Wir werden später zeigen, dass das in der geringen Concentration des Alkalis seinen Grund hatte. Die Wirkung des Salzes ist also an einen bestimmten Alkaligehalt der Küpe gebunden, ober-

Tabelle VI.

\begin{tabular}{|c|c|c|c|c|c|c|c|c|c|c|c|}
\hline \multirow{3}{*}{$\begin{array}{l}\text { No. der } \\
\text { Vers. }\end{array}$} & \multirow[b]{3}{*}{$\begin{array}{c}\text { Wolle } \\
\text { B } \\
\end{array}$} & \multirow{3}{*}{$\begin{array}{c}\text { Vol. der } \\
\text { Firbe- } \\
\text { kitpe } \\
\text { ecm } \\
\end{array}$} & \multirow{3}{*}{$\mid$\begin{tabular}{c|} 
Zusatz \\
v. Chlor- \\
natrinm \\
$2 \mathrm{zu}$ \\
100 comm \\
Furbe- \\
kupo \\
B
\end{tabular}} & \multicolumn{4}{|c|}{$100 \mathrm{ccm}$ Fārbeküpe $\left.{ }^{36}\right)$ enthalten: } & \multirow{3}{*}{\begin{tabular}{|c} 
dug der \\
Ge- \\
nammit- \\
kupe \\
aufge- \\
nomme- \\
ner In- \\
digo \\
B
\end{tabular}} & \multirow{3}{*}{$\begin{array}{c}\text { Verbait- } \\
\text { niss der } \\
\text { aufge- } \\
\text { nomme- } \\
\text { nen } \\
\text { Mengen }\end{array}$} & \multirow{3}{*}{$\begin{array}{c}\text { Indigo } \\
\text { staf der } \\
\text { Wolle in } \\
\text { Proc. } \\
\text { des } \\
\text { Wollge- } \\
\text { wlehtes }\end{array}$} & \multirow{3}{*}{$\begin{array}{l}\text { Ausselen der gefarbter } \\
\text { Woile }\end{array}$} \\
\hline & & & & \multicolumn{2}{|c|}{ Indlgwelss } & \multicolumn{2}{|c|}{ Natrlumbydroxyd ${ }^{\text {36) }}$} & & & & \\
\hline & & & & $\mid \begin{array}{c}\text { Vor dem } \\
\text { Firbon } \\
\text { B }\end{array}$ & $\begin{array}{c}\text { Nach d. } \\
\text { Firben } \\
\text { B }\end{array}$ & $\begin{array}{c}\text { Vor dom } \\
\text { Furben } \\
\text { g }\end{array}$ & $\mid \begin{array}{c}\text { Nach d. } \\
\text { Farben } \\
\text { g }\end{array}$ & & & & \\
\hline $\begin{array}{l}20 \\
21 \\
22 \\
23\end{array}$ & $\begin{array}{l}15,8 \\
15,8 \\
15,8 \\
15,8\end{array}$ & $\begin{array}{l}800 \\
800 \\
800 \\
800\end{array}$ & $\begin{array}{l}0,073 \\
0,148 \\
0,211\end{array}$ & $\begin{array}{l}0,1193 \\
0,1117 \\
0,1178 \\
0,1217\end{array}$ & & $\begin{array}{l}0,0470 \\
0,0475 \\
0,0538\end{array}$ & $\begin{array}{l}0,0438 \\
0,0460 \\
0,0437 \\
0,0451\end{array}$ & & & $\begin{array}{l}0,90 \\
1,00 \\
1,20 \\
1,30\end{array}$ & $\begin{array}{l}\text { mittelblau } \\
\text { etwas dunkler } \\
\text { noch dunkler } \\
\text { noch dunkler }\end{array}$ \\
\hline $\begin{array}{l}24 \\
25 \\
26 \\
27\end{array}$ & $\begin{array}{l}15,0 \\
15,0 \\
15,0 \\
15,0\end{array}$ & $\begin{array}{l}790 \\
790 \\
790 \\
790\end{array}$ & $\begin{array}{l}\overline{0,256} \\
0,762 \\
1,800\end{array}$ & $\begin{array}{l}0,0755 \\
0,0712 \\
0,0732 \\
0,0738\end{array}$ & $\begin{array}{l}0,0597 \\
0,0525 \\
0,0467 \\
0,0472\end{array}$ & 0,0343 & 0,0314 & $\left|\begin{array}{l}0,1248 \\
0,1477 \\
0,2093 \\
0,2101\end{array}\right|$ & $\begin{array}{l}100 \\
118 \\
167 \\
168\end{array}$ & $\begin{array}{l}0,54 \\
0,78 \\
0,92 \\
1,10\end{array}$ & $\begin{array}{l}\text { hellblau } \\
\text { dunkler } \\
\text { noch dunkler } \\
\text { noch dunkler }\end{array}$ \\
\hline $\begin{array}{l}\left.28^{38}\right) \\
29 \\
30 \\
31\end{array}$ & $\begin{array}{l}15,1 \\
15,1 \\
15,1 \\
15,1\end{array}$ & $\begin{array}{l}800 \\
800 \\
800 \\
800\end{array}$ & $\begin{array}{l}\overline{0,244} \\
0,722 \\
1,705\end{array}$ & $\begin{array}{l}0,0521 \\
0,0603 \\
0,0535 \\
0,0609\end{array}$ & $\begin{array}{l}0,0450 \\
0,0459 \\
0,0385 \\
0,0398\end{array}$ & 0,1290 & 0,1179 & $\begin{array}{l}0,0568 \\
0,1152 \\
0,1200 \\
0,1688\end{array}$ & $\begin{array}{l}100 \\
202 \\
211 \\
297\end{array}$ & $\begin{array}{l}0,38 \\
0,48 \\
0,67 \\
0,95\end{array}$ & $\begin{array}{l}\text { hellblau } \\
\text { dunkler } \\
\text { noch dunkler } \\
\text { noch dunkler }\end{array}$ \\
\hline $\begin{array}{l}32 \\
33 \\
34 \\
35\end{array}$ & $\begin{array}{l}15,0 \\
15,0 \\
15,0 \\
15,0\end{array}$ & $\begin{array}{l}800 \\
800 \\
800 \\
800\end{array}$ & $\begin{array}{l}\overline{0}, 256 \\
0,758 \\
1,807\end{array}$ & $\begin{array}{l}0,2069 \\
0,2069 \\
0,2112 \\
0,2110\end{array}$ & $\begin{array}{l}0,1878 \\
0,1822 \\
0,1712 \\
0,1506\end{array}$ & 0,337 & & \begin{tabular}{|l|}
0,1528 \\
0,1976 \\
0,3200 \\
0,4832
\end{tabular} & $\begin{array}{l}100 \\
129 \\
209 \\
306\end{array}$ & $\begin{array}{l}1,05 \\
1,25 \\
1,45 \\
2,12\end{array}$ & $\begin{array}{l}\text { mittelblaq } \\
\text { kaum dunkler } \\
\text { etwas dunkler } \\
\text { dunkler und streifig }\end{array}$ \\
\hline $\begin{array}{l}36 \\
37 \\
38 \\
39\end{array}$ & $\begin{array}{l}30,0 \\
29,9 \\
30,1 \\
30,0\end{array}$ & $\begin{array}{l}1800 \\
1800 \\
1800 \\
1800\end{array}$ & $\begin{array}{l}\overline{0,032} \\
0,073 \\
1,800\end{array}$ & $\begin{array}{l}0,1070 \\
0,1074\end{array}$ & & $\left.0,0057^{36}\right)$ & & & & $\begin{array}{l}0,59 \\
0,56 \\
0,54 \\
0,78\end{array}$ & $\begin{array}{l}\text { hellblau } \\
\text { hellblau } \\
\text { hellblau } \\
\text { etwas dunk ler, streifig }\end{array}$ \\
\hline $\begin{array}{l}40 \\
41 \\
42\end{array}$ & $\begin{array}{l}30,0 \\
29,9 \\
29,9\end{array}$ & $\begin{array}{l}1800 \\
1800 \\
1800\end{array}$ & $\begin{array}{l}\overline{0}, 073 \\
1,800\end{array}$ & $\begin{array}{l}0,1324 \\
0,1322 \\
0,1334\end{array}$ & & $\left.0,0187^{36}\right)$ & & & & $\begin{array}{l}1,50 \\
1,66 \\
2,66\end{array}$ & $\begin{array}{l}\text { mittelblau } \\
\text { etwas dunkler } \\
\text { erheblich dunkler }\end{array}$ \\
\hline
\end{tabular}

In Versuchen 36-42 wurde der Einfluss von Kochsalz auf Hydrosulfitküpe geprüft, und zwar konnte hier der Alkaligehalt bis

36) Küpen $20-35$ enthielten nur Indigweissnatrium ohne Hydrosulfit, $36-42$ waren Hydrosultitküpen. Ansalz der letzteren siehe Anm. 19. Die für 36-42 aufgeführten Alkalimengen geben nur den zugesetzten Überschuss an, der wirkliche Atznatrongehalt war wegen der Gegenwart des Hydrosulfits nicht bestimmbar. Bei den úbrigen Küpen schliessen die aufgeführten Alkalimengen das an Indigweiss gebundene Natriumhydroxyd ein.

37) Bei Versuchon 29, 30, 31, 33, 34, 35 wurde auch nach dom Fārben der Salzgehalt bestimmt. Es fanden sich successive: 0,$250 ; 0,732 ; 1,710$; 0,$256 ; 0,762 ; 1,805 \mathrm{~g}$. Die Zahlen sind etwas höher als die vor dem Färben erhaltenen, was oben (s. Tab. V) erklärt wurde.

38) Identisch mit Versuch 4. halb. sowohl wie unterhalb desselben schwächt sie sich ab.

Die analytische Controle der besprochenen Ergebnisse geschah zunächst durch Analysiren der Küpen nach dem Färben. Daraus berechnen sich die Mengen des "aus der Gesammtküpe aufgenommenen Indigos" und in der darauf folgenden Columne ihre Verhältnisswerthe. Wie betreffenden Zahlen zeigen ein stetiges Ansteigen mit Zunahme des Salzes und der Natronlauge. Das Nachlassen der Salzwirkung bei einer gewissen Höhe der Alkaliconcentration (Versuche 32-35) ist hier nicht zu bemerken. $\mathrm{Da}$ dennoch die Ausfärbungen in diesen Fällen mit steigendem Salzgehalt sich nur wenig vertieften, so folgt daraus, dass ein entsprechender Theil beim Waschen von der Faser wieder abfiel. 
Ferner wurde die gefärbte Wolle jedesmal mit Eisessig extrahirt. Die Methode lieferte Werthe, die im Allgemeinen mit dem Aussehen der Färbung übereinstimmten. Feinere Differenzen aber - wie den oben erläuterten Unterschied zwischen Serie 32-35 und den vorhergehenden - zeigte sie nicht an (vergl. Anm. 20). Das änderte sich nuch nicht durch Bestimmung des Reingehaltes des abgezogenen Indigos ${ }^{33}$ ). Für den vorliegenden $\mathrm{Z}$ weck ist also die Faseranalyse nicht genau genug. Wir messen deshalb ibren Ergebnissen wenig Werth bei und haben sie demgemäss nur kurz in der Tabelle aufgeführt. heisser Natronlauge lōst, wie es bei den Versuchen 3, 7, 8, 20-27 geschehen war, so bleibt die Flüssigkeit stets stark alkalisch. Dagegen findet eine vollkommene Absättigung des Indigweiss statt, wenn man nach der Vorschrift B. A. S. F. eine Stammküpe in folgenden Verhältnissen ansetzt:

$133 \mathrm{~g}$ Indigweisspaste 15 Proc. $=20 \mathrm{~g}$ Indigweiss) werden mit $8,26 \mathrm{ccm}$ Natronlauge $40^{\circ}$ Bé. (=3,99 g Ätznatron) und $60 \mathrm{ccm}$ Natriumhydrosulfitlösung erwärmt, bis vollkommene Lösung erfolgt ist. Das Ganze wird auf $250 \mathrm{ccm}$ aufgefüllt. Eine solche Lōsung enthält nur etwa $2 / 3$ derjenigen Alkali-

\begin{tabular}{|c|c|c|}
\hline \multirow[b]{2}{*}{$\begin{array}{l}\text { No. des } \\
\text { Ver- } \\
\text { suches }\end{array}$} & \multicolumn{2}{|c|}{ In $100 \mathrm{ccm}$ Ktipe } \\
\hline & $\begin{array}{c}\text { Indig } \\
\text { weiss } \\
\text { f }\end{array}$ & $\begin{array}{c}\text { Na OII } \\
g\end{array}$ \\
\hline $43-45$ & 0,02 & 0,004 \\
\hline 46,47 & $\begin{array}{l}\text { 7weite } \\
\text { frische } \\
\text { aus d } \\
\text { K }\end{array}$ & $\begin{array}{l}\text { Zug mit } \\
\text { Wolle } \\
\text { selben } \\
\text { e }\end{array}$ \\
\hline $48-50$ & 0,1 & 0,02 \\
\hline 51,52 & $\begin{array}{l}\text { Zuveite } \\
\text { frische } \\
\text { aus d } \\
\\
\mathrm{K}\end{array}$ & $\begin{array}{l}\text { Zug mit } \\
\text { Wolle } \\
\text { selben } \\
\text { ee }\end{array}$ \\
\hline
\end{tabular}

Tabelle VII.
Salzfrei
1,8 Proc. $\mathrm{Na} \mathrm{Cl}$
5 Proc. $\mathrm{Na} \mathrm{Cl}$

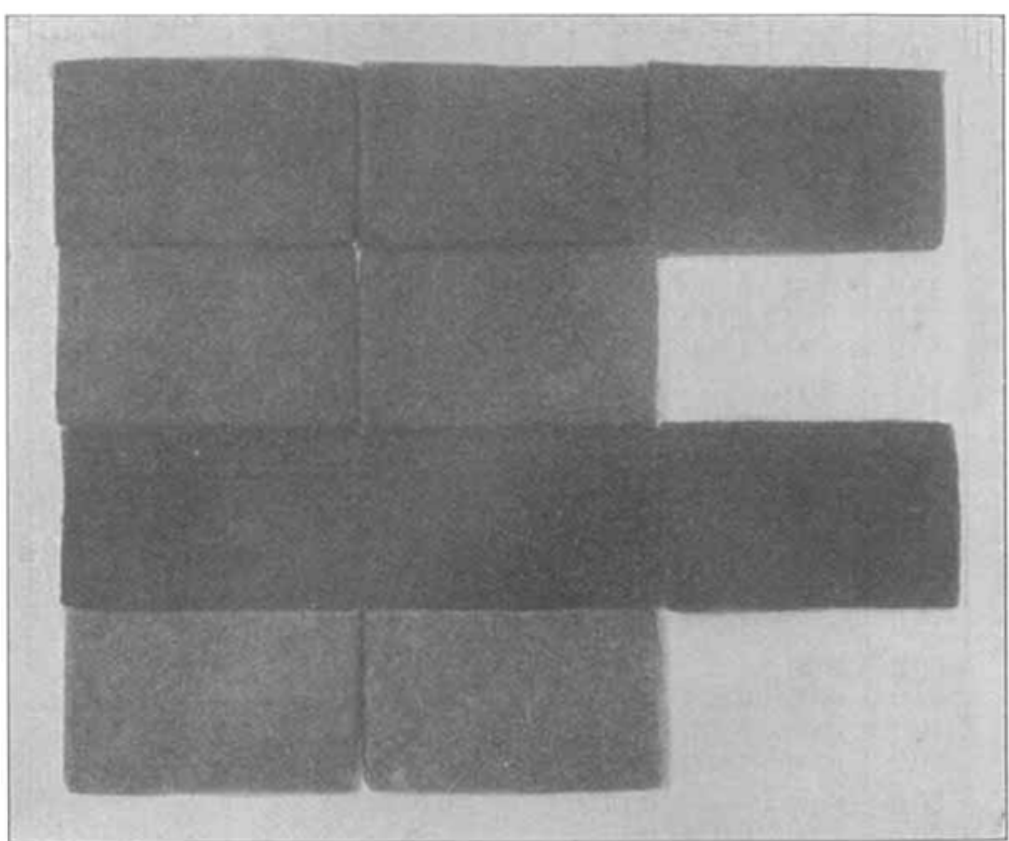

Bei einer Controle der in Tabelle VI niedergelegten Resultate durch Versuche in grösserem Maassstabe zeigten sich dieselben Erscheinungen, die soeben beschrieben wurden; zugleich aber wurde in Verfolg der Versuche 36-39 gefunden, dass bei einer Verminderung des Alkaligehaltes unter das bisber angewandte Mass die Wirkung des Salzes vollkommen verschwindet.

Die bisherige Versuchsanordnung liess diese Thatsache nicht hervortreten, denn wenn man Indigweiss in sehr verdünnter

59) Beim Sulfuriren und Titriren mit Hydrosulfit ergaben sich

bei Nummer:

$\begin{array}{lllll}24 & 32 & 33 & 34 & 35\end{array}$

Procent reines Indigotin: 94,9 89,3 89,5 91,8 94,7

$\begin{array}{llllll}\text { bei Nummer: } & 36 & 39 & 40 & 41 & 42\end{array}$

Procent reines Indigotin: 90,0 91,2 88,6 81,0 97,1 menge, die sich für neutrales Indigweissnatrium berechnet ${ }^{+0}$ ).

Derartige Küpen und bestimmte ihnen mit der Pipette entnommene Theile wurden theils mit, theils ohne Salz zu je 10 Liter Wasser von $50^{\circ}$ gegeben, welches vorher mit $10 \mathrm{ccm}$ Hydrosulfit von Sauerstoff befreit worden war. Die so hergestellten Färbeküpen standen in einem grossen mit Dampf geheizten Wasserbad und dienten zum Färben von je $30 \mathrm{~g}$ loser gewaschener Wolle. Diese befand sich in siebartig durchlöcherten Kästen,

40) Eine genaue Gehaltsangabe ist wegen der Anwesenheit des Hydrosulfits nicht möglich (s. die Einleitung). - Das Hydrosulfit wurde nach der Vorschrift No. 1030 der Badischen Anilin-und Soda-Fabrik aus $10 \mathrm{~kg}$ festem Natriumhydrosulfit, 50 Liter Wasser und 1 Liter Natronlauge $40^{\circ}$ Bé. hergestellt. 
die in die Küpe eingesenkt wurden. Dauer des Küpens (ausser bei 51 und 52) ${ }_{12}^{1 /}$ Stunde, Temperatur $50^{\circ}$.

Die Resultate sowie die Einzelheiten der Versuchsbedingungen ${ }^{41}$ ) ergeben sich aus allerdings gehen feinere Unterschiede bei der Reproduction verloren. Immerhin aber ist diese Methode der Wiedergabe der Resultate besser als die durch Beschreibung oder Faseranalyse wie in Tabelle VI.

Tabelle VIII.

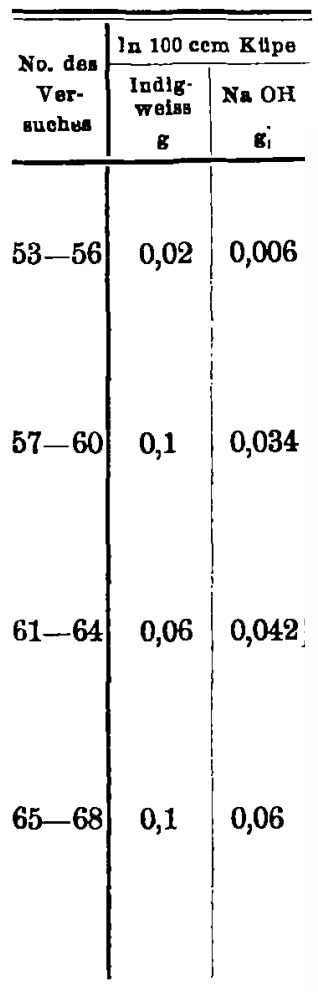

Salzfrei

0,07 Proc.

0,70 Proc.

1,80 Proc. $\mathrm{Na} \mathrm{Cl}$

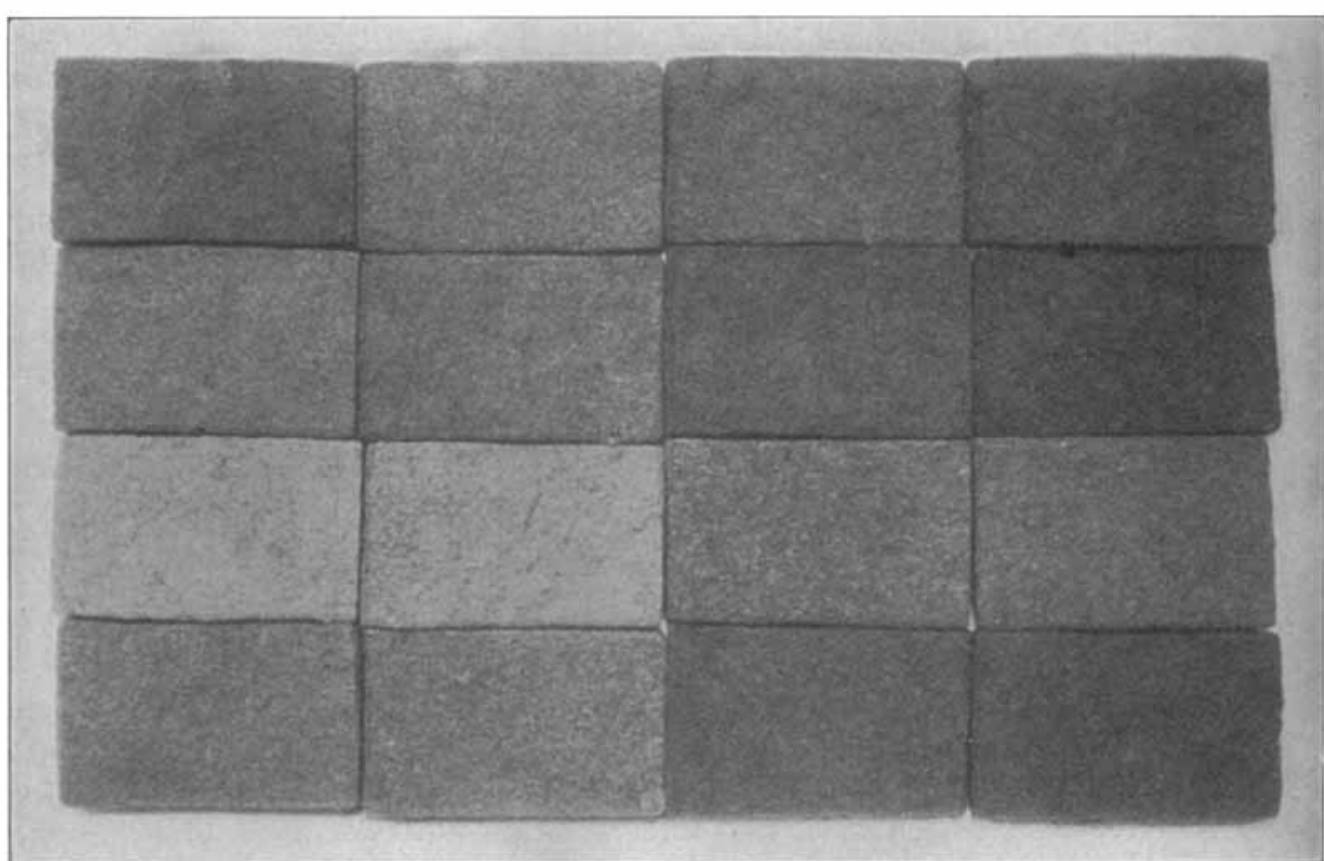

Tabelle IX.

\begin{tabular}{c|c|c}
\hline \hline $\begin{array}{c}\text { No. des } \\
\text { Ver } \\
\text { uuches }\end{array}$ & $\begin{array}{c}\text { In 100 ecm Ktpe } \\
-\begin{array}{c}\text { lndig- } \\
\text { weitu } \\
g\end{array}\end{array}$ & $\begin{array}{c}\text { Na OR } \\
\text { g }\end{array}$ \\
\hline $69-72$ & 0,15 & 0,129 \\
$73-76$ & 0,20 & 0,337 \\
& &
\end{tabular}

Tabelle VII, welche die Ausfärbungen nach photographischen Aufnahmen zeigt. Man erhält daraus einen annähernd richtigen Eindruck von den Abstufungen im Farbenton,

41) Die Zahlen der Tabelle geben nur das zur Stammkūpe zugesetzte freie Alkali, nicht das des Hydrosulfits, welches im Vergleich zu ersteren gering ist und sich leicht aus den Angaben in Anm. 40 berechnen lässt.
Aus dem Vergleich der beiden ersten Färbeproben (No. 43 und 44) ergiebt sich, dass $1,8 \mathrm{~g} \mathrm{Salz}$ in $100 \mathrm{ccm}$ Küpe - dieselbe Menge, die in Versuchen 27, 31 und 35 die Tiefe der Ausfärbungen verdoppelt hatte bei dem hier gewählten niedrigeren Alkaligehalt der Küpe vollkommen unwirksam war. Die Färbungen sind identisch. Sogar 5 Proc. Salz (No. 45) hatten kaum eine wahrnehmbare Vertiefung zur Folge. 
Das Ausbleiben der Salzwirkung könnte man darauf zurückfülıren, dass bei einer Concentration des Farbstoffes von nur 0,02 Proc. die Küpen vielleicht vollkommen erschöpft wurden, gleichgültig ob Salz zugegen war oder nicht, und dass deshalb durch Zusatz des Salzes natürlich auch keine Änderung in dem färberischen Resultat eintreten konnte.

Diese Folgerung wäre irrig, denn die Küpen waren durchaus nicht erschöpft. Ein zweiter Zug aus ibnen (46 und 47) lieferte Ausfürbungen, die fast noch gerade so kräftig waren als die ersten. Auch diese Färbeproben waren unter sich gleich.

Es liegt ferner der Gedanke nahe, dass der Salzzusatz deshalb keine Folgen hatte, weil erfahrungsgemäss quantitative Aussalzung von Farbstoffen in vielen Fällen nicht stattfindet; bäufig bleibt eine Spur in Lösung, und dieser Spur - so könnte man folgern entspricht ein Indigweissgehalt von 0,02 Proc., so dass darum die Anwesenheit des Salzes die Löslichkeit des Farbstoffes nicht herabsetzte und dieser demgemäss auch nicht stärker von der Faser adsorbirt wurde.

Aber auch dieser Einwand ist hinfällig. Versuche 48-50 (Küpzeit $30 \mathrm{Min}$.) und 51 und 52 (Küpzeit $2 \mathrm{Min}$.) zeigen die Ergiebigkeit von Färbeküpen mit und ohne Salz, zu denen fünfmal so viel Stammküpe verwandt worden war wie vorher. Auch hier waren die Ausfärbungen derselben Horizontalreihe identisch. Geringere Indigomengen als die in diesen Küpen lassen die Kochsalzwirkung deutlich hervortreten, falls mehr Alkali zugegen ist. Das hatten Versuche 24-31 gezeigt, und eine Bestätigung ergab sich durch eine neue Versuchsreihe (Tabelle VIII), in der dieselben Alkali- und Salzmengen gewählt wurden wie in 20-27.

Die Färbeküpen in dieser Versuchsreihe wurden wie bei 43-52 hergestellt, dazu kamen abgemessene Mengen Natronlauge.

Ein deutliches Einsetzen der Salzwirkung zeigt sich erst bei einer gewissen Grenze der Alkaliconcentration, die in $53-56$ eben erreicht ist. Denn diese vier Ausfärbungen waren im Original fast identisch. Nur die letzte war eine Spur tiefer als die vorigen. Dagegen bewirkte bei 0,034 Proc. Ätzaatron schon 0,07 Proc. Salz eine merkliche Vertiefung der Nuance (Versuch 58) - die Photographie giebt diese Feinheit leider nicht wieder und das schreitet fort, bis bei 1,8 Proc. Salz ein sattes Dunkelblau erreicht ist, das auf dem Bilde deutlich hervortritt.

Dasselbe zeigen Versuche $61-68$.

Fährt man nun mit der Alkalizugabe fort (Tabelle IX), so wird ebenso wie bei Versuchen 32-39 die Salzwirkung schwächer.
Zwar blieben die Endfärbungen (72 und 76), die bei Gegenwart von 1,8 Proc. Salz hergestellt waren, stets dunkler als die ursprünglichen (69 und 73). Aber eine deutliche Vertiefung der Nuance bei 0,07 Proc. Salz wie in Tabelle VIII liessen Versuche 70 und 74 nicht erkennen. In der letzten Reihe war auch die dritte Ausfärbung nur wenig dunkler als die erste.

Schon bei der Untersuchung der Baumwollküpe (Versuch 6) wurde darauf hingewiesen, dass nicht nur Chlornatrium, sondern auch andere Neutralsalze den Färbeprocess beeinflussen können. Das gilt auch für die Wollküpe. Je 10 Liter Färbekūpe wurden mit folgenden Zusätzen beschickt:

In $100 \mathrm{~cm}$ befanden sich:

\begin{tabular}{c|c|c|c}
\hline $\begin{array}{c}\text { No. d. } \\
\text { Vers. }\end{array}$ & $\begin{array}{c}\text { Indig- } \\
\text { welss } \\
\mathbf{g}\end{array}$ & $\begin{array}{c}\text { Aetz- } \\
\text { natron } \\
\mathbf{g}\end{array}$ & $\mathbf{S a l z}$ \\
\hline 77 & $\mathbf{0 , 0 2}$ & 0,004 & $\mathbf{8}$ \\
$\mathbf{7 8}$ & 0,02 & 0,004 & $\mathbf{4 , 4 5}$ Glaubersalz \\
$\mathbf{7 9}$ & $\mathbf{0 , 0 2}$ & 0,004 & $\mathbf{3 , 8 2}$ Natriumthiosulfat \\
$\mathbf{8 0}$ & 0,1 & 0,034 & - \\
$\mathbf{8 1}$ & 0,1 & 0,034 & 4,45 Glaubersalz \\
$\mathbf{8 2}$ & $\mathbf{0 , 1}$ & 0,034 & $\mathbf{3 , 8 2}$ Natriumthiosulfat
\end{tabular}

Die Mengen der krystall wasserhaltigen Salze sind 1,8 Proc. Chlornatrium äquivalent. Die Concentrationen des Indigweiss und des Alkalis waren dieselben wie bei $43-47$ und $57-60$.

Demgemäss trat ganz das erwartete Resultat ein: Ausfärbungen 77-79 waren identisch, dagegen zeigten 81 und 82 ein doppelt so tiefes Blau wie 80 .

Die Färbeproben sind der Raumersparniss halber hier nicht photographisch wiedergegeben, da sie genau so aussahen, wie die entsprechenden in Tabellen VII und VIII.

\section{Zusammenfassung und Discussion} der Resultate.

Die experimentellen Ergebnisse dieser Arbeit sind folgende:

1. Durch Steigerung des Alkaligehaltes einer Küpe nimmt die Intensität der Ausfärbung ab, was beim Küpen der Wolle mehr zu Tage tritt als bei der Baumwollküpe. Diese qualitativ bereits bekannte Erscheinung wurde quantitativ verfolgt.

2. Durch Zusatz von Kochsalz, Glaubersalz und Natriumthiosulfat findet keine Vertiefung der Nuance statt, wenn die Küpe nur so viel Natronlauge enthālt, als zur Lösung des Indigweiss erforderlich ist. Dagegen werden bei sehr geringem Alkaliüberschuss die Ausfärbungen durch Zu- 
geben von Salzen intensiver, und zwar bewirkt 1,8 Proc. Kochsalz in der Wollküpe eine Verdoppelung der von der Faser fixirten Menge. Hierbei wird das Salz selber nicht von der Faser adsorbirt.

3. Auch bei weiterer Steigerung der Alkaliconcentration, bis zu etwa 0,3 Proc. wird die Küpe durch Salzzusatz mehr erschöpft als ohne diesen. Die Menge des fixirten Farbstoffes wächst aber nicht in der entsprechenden Weise, so dass die Wirkung des Salzes auf die Intensität der Ausfärbung weit geringer ist als bei niedrigerem Alkaligehalt.

Die Wirkung eines Neutralsalzes, wie sie unter 2 und 3 geschildert ist, hat man bis jetzt in der Färbereitechnik nicht beobachtet. Allerdings weiss man, dass Salze die Löslichkeit der Farbstoffe herabsetzen können und diese dann mit erhöhter Intensität auf die Faser ziehen, und da diese Erscheinung für das Färben der ungebeizten Baumwolle mit substantiven Farbstoffen von höchster Bedeutung ist, so gehört sie zu den beststudirten auf färbereichemischem Gebiete. Auch findet bei solchen Operationen häufig ein Zusatz alkalisch wirkender Salze oder von Natriumhydroxyd statt, es fehlt also scheinbar nicht an Vergleichsfällen für die Wirkungsweise von Salzen in der Küpe. Trotzdem ist nuch kein Fall bekannt geworden, wo es von der Anwesenheit geringer Alkalimengen abhängt, ob das Salz den Färbeprocess beeinflusst oder nicht. Dieses Kommen und Gehen der Salzwirkung bei minimalen Änderungen der Alkalität der Küpe kann geradezu als ein färberisches Paradoxon bezeichnet werden.

Wie ist diese Erscheinung zu deuten? Die Möglichkeit einer Erklärung ergeben die Zahlen der Versuche 45-76, wenn man aus ihnen das moleculare Verhältniss von Indigweiss und Natriumhydroxyd berechnet ${ }^{42}$ ).

Es kamen auf 1 Molecül Indigweiss

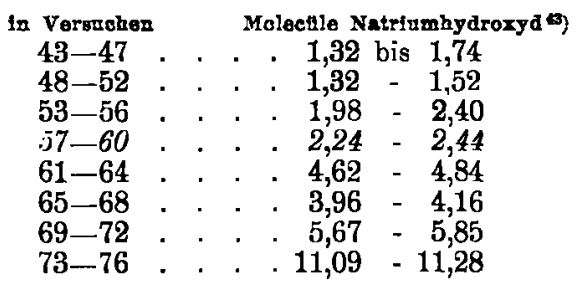

12) Eine genaue Berechnung ist nicht möglich, da man nicht weiss, wie viel Alkali durch etwaige Oxydation des Hydrosulfits absorbirt wurde. Diese Fehlerquelle ist indessen im vorliegenden Falle jedenfalls unbedeutend, da alle Küpen frisch waren und höchstens soviel Hydrosulfit oxydirt sein konnte, wie dem Luftgehalt des verwandten $\mathbf{W}$ assers entsprach.
Die Zahlen zeigen, dass die Küpen 43-52 zum Theil jedenfalls saures Indigweissnatrium enthalten mussten; von Versuch 53 ab war die Möglichkeit zur vollkommenen Absättigung des Indigweiss in Form seines neutralen Natriumsalzes gegeben.

Das Entstehen dieser beiden Salze in verdünnten Lösungen erfordert, wie man annehmen muss, einen gewissen die $\mathrm{Hydro-}$ lyse zurückdrängenden Überschuss an Alkalị. Es ist aus diesem Grunde denkbar, dass bei Versuchen 43 - 56 die Küpen zum grössten Theil saures Indigweissnatrium enthielten und dass erst bei Versuchsreihe 57-60 vollkommene Absättigung des Indigweiss erfolgt war. Gerade bei dieser Serie (die durch Kursivdruck hervorgehoben ist) machte sich der Einfluss des Chlornatriums geltend, vorher zeigte er sich in deutlicher Weise nicht; nur bei 56 war er eben bemerkbar.

Es lässt sich also ein Zusammenhang zwischen der Anwesenheit von neutralem Indigweissnatrium in der Küpe und der Wirkung des Kochsalzes vermuthen. Man kann sich vorstellen, dass saures und neutrales Indigweissnatrium in ihrer Löslichkeit einen ähnlichen Unterschied aufweisen, wie saures und neutrales Calciumcarbonat ${ }^{44}$ ), und dass anorganische Salze die Ausfärbungen dann vertiefen, wenn die schwerer lösliche Indigweissverbindung vorhanden ist.

Diese Annahme ist bis jetzt eine rein hypothetische, da man über die betreffenden Indigweisssalze sowie über ihre Löslichkeit und den Grad ihres hydrolytischen Zerfailes nichts weiss.

Dagegen steht ein Versuch zur Deutung des Abfalles der Salzwirkung bei allzu grosser Alkaliconcentration auf etwas festerem Boden.

Verschiedene Thatsachen machen es wahrscheinlich, dass in der Wollküpe nicht Indigweissalkali das färbende Princip ist, sondern Indigweiss in freiem Zustande, welches durch Hydrolyse aus seinem sauren oder neutralen Salz entstanden ist. Es geht das erstens daraus hervor, dass man Wolle bei $50^{\circ}$

43) Die Berechnung geschah in zweifacher Weise. Als unterste Grenze der Alkaliconcentration wurden die in Tabellen VII, VIII und IX verzeichneten Zahlen angenommen. Die oberste ergab sich, indem ausser ibnen wouh der Alkaligehalt des Hydrosulfits, und zwar der zur Stammküpe und zum Verschärfen der Färbekūpen gegebenen Mengen (sieho oben), in Rechnung gezogen wurde. Àf diese Weise entstanden die beiden Zahlenreihen, deren Werthe übrigens nur wenig von einander verschieden sind, da die Menge des Hydrosulfits gering war.

44) Selbstverständlich soll mit dieser Parallele nicht gesagt sein, dass Indigweissnatrium gerade so wenig löslich ist wie kohlensaurer Kalk. 
küpen muss ${ }^{45}$ ), also bei einer Temperatur, die die Hydrolyse erleichtert. Ferner giebt es thatsächlich technische Verfahren ${ }^{46}$ ) zum Färben mit freiem Indigweiss.

Beruht nun wirklich darauf der Färbeprocess in der Wollküpe ${ }^{+7}$ ), so ist es ver-

15) Vergl. Anm. 3.

45) Kallab, Deutsches Wollengewerbe 1896, 38, S. 624. Patentanmeldung F. 12826 IV $/ 8 \mathrm{k}$ der Höchster Farbwerke.

17). Die Anregung zu dieser Auffassung des Färbeprocesses in der Wollküpe gab uns ein Schreiben des Berrn Prof. v. Georgievies vom 22. Mai 1900.

Wir hatten in unserer dritten Mittheilung eino Āusserung dieses Forschers citirt, in der eine chomische Vereinigung des Indigweiss mit der Faser angenommen wird. Herr v. Georgievies hatte die Freundlichkeit, uns daraufhin Folgendes zu schreiben:

„. . . Eine chemische Vereinigung des Indigweiss mit der Cellulose der Baumwollfaser hatte ich zu einer Zeit angenommen, in welcher meine Arbeiten über das Wesen des Färbeprocesses noch nicht begonnen waren. Heute stohe ich auf dem Standpunkt, dass man in keinem Falle - also auch nicht in dem vorliegenden - berechtigt ist, eine chemische Vereinigung des Farbstoffs mit der Substanz der betreffenden Faser beim Färben anzunehmen.

Eine Adsorption des Indigweiss in Salzform halte ich für unwahrscheinlich. Dass beim Färben auf der Küpe auch Alkali neben Indigweiss ron der Baumwolle aufgenommen wird, ist gewiss richtig. In der Schafwollfärberei z. B. ist es schon lange bekannt, dass man einen zu grossen Kalkgehalt der Küpe durch Einblauen von Wolle vermindern kann; ich kann mir aber ganz gut denken, dass Alkali und Indigweiss beide unabhängig von einander adsorbirt werden, etwa so wie Schwefelsâure und Thonerde beim Liegenlassen von Schafwolle in einer Lösung von Ảluminiumsulfat. In diesem Fall habe ich nachgewiesen, dass je nach der Concentration der Lōsung entweder mehr Thonerde oder mehr Schwefelsäure aufgenommen wird. .... Man hat sich demnach Indigweisskalk als eine in der Küpe hydrolytisch zerlegte Verbindung vorzustellen, wodurch die auch von Ihnen constatirte Thatsache, dass ein Überschuss von Alkali in der Küpe die Indigoaufnahme vermindert, erklärlich erscheint.“

Wir betrachteten in unserer früheren Arbeit (diese Zeitschr. 1900, 418) den Küpprocess als eine Aufnahmo von Indigweissnatrium durch die Baumwolle, und was die Wollküpe betrifft, so gelang es uns nicht, mit einer wāssrig-alkoholischen Lōsung von Indigweiss Wolle auch nur einigermaassen reibecht anzufärben. Nach dem oben Mitgetheilten sehen wir in diesem Versuch keinen Einwand mehr gegen die Annahme, dass sich in der Wollküpe freies Indigweiss mit der Faser vereinigt, da mōglicherweise die Gegenwart des Alkohols cine zu rasche Oxydation veranlasst und dadurch die innige Vereinigung feinster Farbstoffpartikel mit der Faser verhindert hatte. Offenbar kommen reibechte Färbungen nur dann zu Stande, wenn zunächst eine innige Vereinigung des Leukoindigos nit der Faser stattgefunden hat und in Folge dessen bei der darauf folgenden Oxydation der lndigo in äusserst feiner Vertheilung in der Faser abgelagert wird. Sobald das geschehen ist, befindet sich nach der bisher geltenden Ansicht der Indigo rein mechanisch, ständlich, warum durch Alkaliüberschuss die Wirkung von Kochsalz abgeschwächt wird.

Zwar veranlasst auch in diesem Falle das Salz eine weitergehende Erschöpfung der Küpe - wie aus den Analysen in Versuchen 32-35 hervorging - indessen kann nur ein Bruchtheil des als Natriumsalz auf die Faser getriebenen Farbstoffes fixirt werden, weil in Folge der hohen Alkaliconcentration der Grad der hydrolytischen Dissociation ein zu geringer ist und der Faser verhältnissmässig wenig freies Indigweiss dargeboten wird.

Was wir hier zur Erklärung des Küpprocesses und der eigenartigen Wirkung anorganischer Salze beibrachten, bedarf noch der experimentellen Bestätigung durch Messung von Löslichkeit und hydrolytischer Dissociation der Indigweissalkalisalze. Die von uns beschriebenen Versuchsergebnisse können also noch nicht als mit Sicherheit erklärt gelten. Dennoch geben sie Aufschluss über die Eingangs aufgeworfenen Fragen.

Was zunächst die schon erwähnte Patentschrift von Gutbier \& $\mathrm{Co}$. angeht, so ist es klar, wie die Erfinder dazu kamen, ihren Patentanspruch auf einen Zusatz von 5 Proc. Chlornatrium und äquivalente. Salzmengen zu beschränken. Offenbar arbeiteten sie zufällig mit einer Küpe von sehr geringem Alkaligehalt und in einem solchen Fall wirken kleine Salzmengen nicht.

In der Praxis sind aber derartige Küpen nicht die Regel. Zwar kommt es niemals vor, dass Wolle bei Gegenwart von so viel Natronlauge geküpt wird, wie in Versuchen 69-76. Dagegen sind Schwankungen wie in 43-64 nicht zu vermeiden, da die Küpe durch Oxydation des Hydrosulfits sich in unberechenbarer Weise der Acidität nähert

in "Juxtaposition" (0. N. Witt, Chem. Technol. d. Gespinnstf. 1891, 374), mit den Faserpartikeln. Demgegenüber möchten wir kurz eine Untersuchung erwähnen, die zur Zeit den Einen von uns (A.B.) in Gemeinschaft mit den Herren Dr. Duntze und Dr. Kufferath beschäftigt und die zur Darstellung einer Reihe wohl charakterisirter Salze des Indigos geführt hat. Der Indigo hat also deutlich basische Eigenschaft und es frägt sich, ob seine Echtheit nicht zam Theil dadurch bedingt wird, dass or sich ähnlich wie andere basische Farbstoffe mit der Wolle verbindet, sobald er durch Oxydation in der Faser entsteht. -

Gegen die Annahme eines Küpprocesses, der mit dem Entstehen von freiem Indigweiss durch Hydrolyse rechnet, könnte man einwenden, dass Indigweiss nach dem, was man bisher von diesem Körper weiss, in Wasser unlöslich ist (vergl. z. B. $\mathrm{Kallab}$ a. a. O.), also durch Hydrolyse von Indigweissnatrium ausfallen müsste. Diese Ansicht ist dabin zu berichtigen, dass Indigweiss sich in siedendem Wasser sichtlich mit gelber Farbe löst, also wahrscheinlich auch bei der Temperatur der Wollkūpe gelöst bleiben kann. 
und darum nach Gutdünken mit Alkali corrigirt werden muss. Es kann dann sehr wohl eintreten, dass viel geringere Salzmengen, als Gutbier sie vorschreibt, die Nuance merklich vertiefen, ebenso gut aber liegt die Möglichkeit vor, dass wie bei Versuchen 45 und 50 die 5 Proc. Chlornatrium des citirten Patentes fast ohne Wirkung $\operatorname{sind}^{48}$.

Für die gewöhnliche Praxis der Hydrosulfitküpe ohne weitere Zusätze ergiebt sich, dass das Natriumbydrosulfit und die aus ihm entstehenden Salze den Verlauf des Färbeprocesses in sprunghafter Weise beeinflussen müssen. Dieser Einfluss bleibt latent, wenn die Küpe kein freies Alkali enthäl, er tritt hervor, sobald letzteres auch nur in geringem Maasse vorwaltet, er verschwindet wieder, sobald das Hydrosulfit zu Pyrosulfit wird und dieses die Natronlauge abstumpft.

Dazu kommt noch die Änderung der Farbnuance durch das Wechseln der Alkalität an und für sich.

Es wird darnach begreiflich, warum es schwer ist, mit der Hydrosulfitküpe auf
Wolle stets gleichbleibende Farbtöne zu erzielen. Gerade das aber ist es, was vom Färber verlangt wird, denn seine Arbeit ist eine Präcisionsarbeit, wie kaum eine zweite in der Technik. Und darum greift er zu irgend einer Methode, von der er glaubt, dass sie keine wechselnden Resultate giebt, sei es auch eine so veraltete wie die Gährungsküpe.

Die im Vorstehenden beschriebenen Ausfärbungen 43-82 wurden in der $\mathrm{Badischen}$ Anilin- und Sodafabrik angefertigt. Wir sprechen dieser Firma für ihr liebenswürdiges Entgegenkommen und ihre werthvolle Förderung der Arbeit durch Rath und That unseren verbindlichsten Dank aus.

Ebenso danken wir Herrn Dr. J. Wagner, Assistenten am chemischen Institut Bonn, welcher die Güte hatte, die photographischen Aufnahmen zu machen, und ferner den Herren Dr. Foerster, Dr. Sieben, Dr. Walter und Cand. chem. Radermacher, die uns in vortrefflichster Weise bei den Versuchen unterstützten.

Bonn, Chenisches Institut der Universität und Siegfeld bei Rolffs \& Co.

\section{[Weitere Vortrüge folgen.]}

\section{Sitzungsberichte.}

Sltzang der Naturforschenden Gesellschaft Basel. Vom 4. Juni 1902.

Die Sitzung der Natarforschenden Gesellschaft Basel vom 4. Juni wurde abgehalten in Gomeinschaft mit der Medicinischen Gesellschaft Basel.

Herr Prof. Dr. Jaquet sprach ūber Apparate zur Messung des Gasst of wechoels des Menschen. An Hand von Ausführungen über

48) Dieses unregelmässige Erscheinen einer Salzwirkung in der Küpe bei Zusatz von geringem Alkaliūberschuss führt zu einer merkwürdigen Modification dessen, was man bisher über den Einfluss des Alkalis in der Küpe annahm. Man glaubte, dass von zwei Küpen mit gleichem Indigogehalt diejenigo am tiefsten färbt, die am wenigsten Alkali enthält. Das ist für salzfreie Kūpen jedenfalls richtig. Nun stelle man sich aber vor, dass eine Küpe etwa so viel freie Natronlauge enthält, wie in Versuchsreibe 57-60 angegeben ist, und zugleich so viel Salz, wie Versuch 60 entspricht. Man denke sich weiter, dass durch Oxydation des Hydrosulfits dieser Alkaliüberschuss verschwindet. Was wird die Folge sein? Einestheils rertieft sich dis erbaltene Ausfürbung in Folge der Alkaliabnahme, zugleich aber wird das Salz, welches bei Gegenwart freier Natronlauge den Farbstoff auf die Faser trieb, jetzt ebenso unwirksam sein, wie in Versuchen 43-52, und die Ausfürbung wird dementsprechend heller. Es ist wohl denkbar, dass dieser Abfall der Farbintensität grösser ist als jene durch Neutralisation des Alkalis betingte Znnahme, und so könnte es kommen, dass in Folge ven $\mathrm{Ab}$ stumpfung der Natronlauge weniger Indigweiss auf die Faser zieht als vorher. die geschichtliche Entwicklung des Respirationsapparates, in denen der Vortragende die verschiedenen vorgeschlagenen Constructionen vorfährte, stellte er die Anforderungen zusammen, die an einen exact wirkenden Respirationsapparat gestellt werden müssen. Etwas eingehender sprach or ron der Zuntz'schen Constraction, die in letzter Zeit am meisten praktische Verwendung gefunden hat. In diesem Apparat kann die Versuchsperson vollkammen frei athmen. Die Kohlendioxydbildang und der Sauerstoffrerbrauch können genau bestimmt werden, was gestattet, den respiratorischen Coëfficienten za berechnen. Ebenso kōnnen dio verschiedenen Phasen eines Versuches verlolgt werden, was wieder einen Vortheil gegenüber den Constructionen von Pettenk ofer und HoppeSeyler bedeutet. Zum Schluss führte der Vortragende den von ihm constrairten Apparat vor, welcher aus einer hellen, vollkommen luftdicht abgeschlossenen Kammor besteht, in welcher dio Versuchsperson bequem sitzen oder liegen kann. Eine Zuleitang von Strassenluft constanter $\mathrm{Za}$ sammensetzung macht die wiederbolte Analyse der eintretenden Luft überflüssig. Eine Pampo entfornt die verbrauchte Laft and fährt dieselbe darch eine Gasuhr. Darch eine sinnreiche Einrichtung wird der Exspirationsluft ror dem Eintritt in die Gasubr der zweitausendste Theil entnommen und in den erhaltenen Proben anf gasanalytischem Wege Sauerstoff und Kohlendioxyd bestimmt. Zur Analyse dieser Luftproben bedient sich der Vortragende der Methode von Petterson, die asusserst 